\title{
New Insights on Stimulating the Lung Meridian Based on Modern Neurophysiology
}

\author{
Peter Chin Wan Fung ${ }^{*}$, Regina Kit Chee Kong ${ }^{2}$ \\ ${ }^{1}$ Division of Medical Physics, Department of Medicine, University of Hong Kong, Hong Kong, China \\ ${ }^{2}$ School of Chinese Medicine, Southern Medical University, Quanzhou, China \\ Email: *peterallegro333@gmail.com
}

How to cite this paper: Fung, P.C.W. and Kong, R.K.C. (2018) New Insights on Stimulating the Lung Meridian Based on Modern Neurophysiology. Chinese Medicine, 9, 75-117.

https://doi.org/10.4236/cm.2018.93006

Received: June 6, 2018

Accepted: July 6, 2018

Published: July 9, 2018

Copyright ( 92018 by authors and Scientific Research Publishing Inc. This work is licensed under the Creative Commons Attribution-NonCommercial International License (CC BY-NC 4.0). http://creativecommons.org/licenses/by-nc/4.0/ cc) (i) (8) Open Access

\begin{abstract}
Chronic obstructive pulmonary diseases (COPD) caused 3.2 million deaths worldwide in 2015 [1]. Therapeutic treatments, including acupuncture \& herbal medicine have been applied to handle this disease with certain efficacies in the domain of traditional Chinese Medicine. However, very few analyses on the mechanisms behind the efficacies can be found in literature. Without understanding the basic mechanisms behind any medical treatment is the bottle-neck to advancement of possibly effective therapy of any kind. Based on this argument, we start off a series of studies on the neurophysiological consequence of acupuncture/acupressure applied to the Lung Meridian. We explain how the sensory signals (by sympathetic nerves) follow the spinothalamic tracts to the thalamus and then to the primary sensory cortex. The neurons of these ascending tracts synapse the motor neurons which activate some of the different organs of the respiratory system-diaphragm, nose, larynx, scalene muscles, trachea, lungs, intercostal and supporting abdominal muscles. The sensory signals at the neo-cortex are then passed on to the motor neurons in the primary motor cortex. The activated neurons project mainly along two descending tracts: anterior and lateral corticospinal tracts. Neurons of these tracts project to activate again some of the respiratory organs, plus the motors neurons related to the digestive system, including the large intestine. On the other hand, an intrinsic, automatic breathing system in the brainstem sends rhythmic signals through the bulbospinal tract system, which contains a special type of neurons-the pre-sympathetic neurons. These neurons, via interneuron relay, synapse motor neurons which mobilize the organs of the respiratory organs to function. Since the "Lung Meridian induced" signals and the intrinsic signals are sent by different types of neurons, we propose that stimulating the Lung Meridian might activate/supplement the action of the intrinsic system during some pathological states. Though the initial suggestion is supported by in vitro/(in vivo) experiments in detailed steps, clinical trials
\end{abstract}


await future development.

\section{Keywords}

Traditional Chinese Medicine, Lung Meridian, Acupuncture, Acupressure, Moxibustion, Spinothalamic, Anterior Corticospinal, Lateral Corticospinal and Bulbospinal Tracts, Respiratory Centers, Pre-Sympathetic Neurons, Chronic Obstructive Pulmonary Diseases, Sleep Apnea

\section{Introduction}

\subsection{Evidence that the Meridians Form a Very Efficient Communication Network of the Body, Particularly When They Are Stimulated}

In a multi-cellular organism, a system of communication must have developed from the mesoderm to cater for the coordination of the function of a group of cells for morphogenic development, growth, wound healing, surveillance. Such a communication system must be able to detect mechanical stimuli even before birth-cells can detect the shear force arising from the flow of embryonic fluid to guide growth. Ample evidences of research in the past two decades point to the recognition of the origin of numerous physiological process-mechanotransduction. This term is defined to be a process via which the cellular response to the mechanical stimuli is converted into biochemical signals to elicit cellular response specific to such stimuli. A cell has mechanical sensors called integrins on its membrane to detect mechanical stimuli. Every cell group/organ (even a single blood vessel, a single nerve fiber) has an extra-cellular matrix (ECM) built of collagen fibers on which proteins named proteoglycans and glycoproteins are attached. Now water is the chief medium of transport in the body; water molecules have polar structure, and form (positive charged) protons/hydronium ions, (negative charged) hydroxyl ions among the water molecules themselves. A water molecule has asymmetrical arrangement of hydrogen atoms, so that "one side" of a water molecule is polarized with net positive charge, whereas the other side has more negative charge field nearby. Referring to the ECM discussed above, some specific proteoglycans are connected chemically to tree-like structure of hyaluronic acid, which is negatively charged on one side of its structure, and is attracting positively charged hydronium ions and water layers. The hyaluronic acid with bound layers of polarized water forms a viscous fluid, called the ground substance. This thick liquid plus some free water forms the interstitial fluid which plays very important physiological roles. The interstitium with the interstitial fluid forms the non-living part of a large communicative network (generally called the fascia) embracing all organs of the body and provides a platform for interactions among "resident cells", "cells in transit", and cell-groups of various organs. This platform is a huge network for communication for transmission of mechanical, electrical and chemical signals throughout the body [2] 
[3]. The following gives concrete evidence that the TCM Meridians form the more effective tracts in the fascia for various forms of communication of information and exchange of energy.

The majority ( $80 \%)$ of the acupoints on the TCM Meridians were identified to be located in one type of connective tissue (such as loose connective tissue) or another (such as the periosteum) [4] [5] [6] [7].

The major "residence cells" in the fascia are fibroblasts, which synthesize collagen fibers, the main constituents of the network. When the Meridians are stimulated by acupuncture/acupressure, the mechanical tensions of the Meridian tracks are enhanced. Now the activity of the fibroblasts is activated by larger mechanical tension, and the Meridians automatically form efficient durotaxis tracts for these fibroblasts [8]. The fibroblasts also play the role of stem cells and can change their phenotypes according to the tensions of the collagen fibers on which they are crawling. For example, they become chondrocytes when they migrate to the neighborhood of the knee where the tension is larger than that in the skin. Fibroblasts become osteocytes when they crawl on the connective tissues of the bone (periosteum). Stimulating the Meridians would thus activate the metabolism and wound healing process; see discussion in [2] [8] [9]. Also, when the Meridians are stimulated mechanically, the mechanical tensions of the collagen fibers are increased, and the fibroblasts are activated to become stellate form, with processes extending in all directions. The fibroblasts themselves become a connected network, with their processes in contact, participating also in transmission of mechanical signals [2] [10].

Moreover, fibroblasts secret protein fibers called fibronectin (Fn). These fibers fold up to spherical shape during the dormant state; however, the activated fibroblasts exert pulling forces on the Fn protein fibers, so that these Fn protein fibers are elongated, connecting the already existing collage fiber network [11]. The density of the collagen-Fn network is then increased significantly.

It is fruitful to learn that when the Fn protein structure is unfolded, a site of Fn, which enables the "docking" of the transformation growth factor- $\beta$ (TGF- $\beta$ ) is then exposed. TGFs are poly-peptides, being secreted by cells in the interstitium. Anchoring of TGFs can help the growth and change in phenotyping of mesenchymal stem cells, which are "transient cells" in the interstitium, and are essential in wound healing [12] [13] [14].

It has been established that the number of mast cells along the Meridians, particularly at acupoints, is significantly larger than that outside the Meridians [2] [15].

Mast cells were only known to be responsible for allergy reaction some two decades ago. However, recent research indicates that mast cells participate in many physiological functions. In particular, mast cells are essential for the growth of fibroblasts (which are the most important cells in the connective tissues) and the mesenchymal stem cells, in the fascia [16] [17] [18]. Another important aspect is that mast cells tend to be interactive with nerve cells at acu- 
points [19]. Moreover, the Primo Vasculature system was found in the liver, large and small intestines, urinary bladder and other parts of rat [20]; such systems with small vessels and nodes were also revealed in seven types of animals and the placenta of human [3] [21]. The density of mast cells in the Primo Vasculature structure of rat was found to be even several times greater than that found at the acupoint ST 36 [15] [20] [22]. It has been inferred by the workers of the Primo Vasculature system that such small ducts, called Bong Han ducts previously, were part of the TCM Meridians. Using "positron induced X-ray emission" technique, it was reported that the density of calcium ions in sites along some foot Meridians was significantly greater than that of non-Meridian sites. Calcium ion is the most important second messenger for numerous physiological functions. Other studies reported similar result [23] [24] [25]. Electrical stimulations at acupoints have long been applied by many groups to treat various diseases, from pain to stroke, with certain efficacies [26] [27] [28] [29]. Moxibustion has a long history in TCM, and we can also treat acupoints with mild hyperthermia and there is evidence that such treatment affects the functions of organs far away from the excitation sites [30] [31].

All these findings, plus others reviewed in [2] [8] [9] indicate convincingly that when the Meridians are stimulated mechanically, electrically, or by heating can be transmitted through the Meridians with better efficiency than non-Meridian tracts. Certainly, the fluids (interstitial fluid, blood, and lymph) play crucial role also in the physiological conditions of the interstitium which can affect the communication of these signals, as explained in detail before [3].

\subsection{Objectives of This Article-Having Established the Platform of Communication, We Proceed to Investigate the Pathways of Communication via Nerves}

For survival of the human body, we know that: 1) oxygen and nutrients must be supplied to all cells forming well-defined organs such as kidney, and the debris of metabolism must be discharged from the body; 2) oxygen and nutrients must also be supplied to "residence cells" and "cells in transit" in the interstitium [2]; 3) Cells in 1) and 2) have to communicate continuously to carry out numerous physiological functions. We have analyzed in detail how an integrative five-fluid system works in performing the duties 1) and 2) [2] [3].

Having understood the basics of the communication platform, it is natural to analyze how do organs/(cells in the interstitium) communicate. In general, we understand that nerve fibers, blood vessels, lymphatic vessels are running in groups or parallel. The density of nerves, blood vessels is higher along the Meridians as reviewed in [2] [8] and [9]. Nerves are playing dominant roles in numerous physiological functions. Now every nerve fiber, every blood vessel is "surrounded' by at least one layer of connective tissue which is mainly built of collagen of one type or another. It has been demonstrated that applying manual acupuncture at an acupoint with either a) twirling and rotation modality, or b) lifting and thrusting modality would enhance the mechanical tension of the col- 
lagen fibers around the site of application (see Figure 2 and Figure 3 of [32] and [33]). The stimulated (free) collagen fibers are connected directly or indirectly to the collagen layers enclosing nerve fibers, blood and lymphatic vessels. There is direct evidence that the effects of needle rotation reached several centimeters [34]. Anatomical analysis showed that $80 \%$ of the acupoints were found amidst the connective tissues [6]. We anticipate that stimulation at the acupoints or along the Meridians would exert mechanical pull on the nerve endings, nerve fibers directly, or indirectly through the linkage between the collagen fibers in the (loose or deep) connective tissues around the application site and the outermost layer embedding the nerve fiber (epineurium in peripheral nerve). Similarly, acupuncture exerts also mechanical pull on the outer layer (adventitia) of a blood vessel. Since there is a numb feeling while acupuncture action is applied, we can ascertain that acupuncture would stimulate the nervous system. Therefore, we follow up the neurophysiological response when an acupoint of the Lung Meridian is stimulated in this first paper of the series. We need to go into much detail, in most cases, to study the function of each responding neuron, in order to investigate in what ways stimulating the Lung meridian can affect the functions of (A) respiratory system, which involves the sub-parts: (A1) diaphragm, (A2) nose, (A3) larynx, (A4) the pharynx (voice box containing vocal cords), (A5) trachea (A6) lungs, (A7) muscles groups that support breathing, (A8) the respiratory centers at the brain stem (pons and medulla oblongata). The function of the respiratory system was considered closely related to that of (B) the large intestine, and (C) the sweating glands of the body over 1600 years ago in the oldest TCM literature. At that time the slightest bit of neurophysiology-neuroanatomy knowledge was yet to be accumulated. In view of the objective mentioned, we will introduce briefly some basics of the nervous system, and the functions of anatomical parts involved in sub-parts of (A), plus the (A-B) connection in this paper. The impact of stimulating the Lung Meridian on the functions of the blood circulation and lymphatic systems is outside the scope of this paper, but the issue will be treated in another paper related to the Lung Sinew channel.

\section{Some Basic Properties of Neurons Discussed in This Paper}

\subsection{Types of Neurons, Sensors of Nerves and the Related Transmission Processes Associated with the Stimulation of TCM Meridians Discussed in This Paper}

The cell body contains the nucleus. If the cell body (perikaryon) has one branch to transmit electrical signals, it is called a unipolar neuron, which is not discussed much in this paper. Figure 1(a) shows the structure of a motor neuron. Since the cell body of a motor neuron has many branches of dendrites around the cell body (yellowish structure with sharp ends) plus an axon (yellowish foot-like structure) extending to target the dendrite or cell body of another neuron, it is called a multipolar neuron [35]. The axon is insulated electrically by sheath segments. 
Motor neurons connect the brain cortex and the spinal cords at different levels. There are also numerous motor neurons exit from the spinal cord, and innervate skeletal muscles, smooth muscle of organs (such as that in the digestive system), and glands throughout the body. The motor neurons therefore have direct voluntary control of the body. Motor neurons have relatively big diameter $\sim 12$ - 20 micro-meters.

Figure 1(b) shows schematically an interneuron, which is shorter in length in the nervous system, and there are two branches coming out of the cell body; the cell is called a bipolar neuron. An interneuron plays the relay role. In the spinal cord, the axon end has been considered as non-myelinated. Recent finding shows that the interneurons in the neocortex (cortex close to the internal part of the scalp) are myelinated [36]. In Figure 1(b), we present only the myelinated type of interneuron. Figure $1(\mathrm{c})$ shows the morphology of a typical pseudo-unipolar neuron. Most sensory neurons are pseudo-unipolar neurons, and some are bipolar neurons. The cell body of a pseudo-unipolar neuron develops a single process which branches into two processes, one giving rise to the dendrites and the other to the axon. The cell body looks like "sticking out" from the elongated cell. The sensory neurons can be heavily myelinated (A- $\beta$ fibers), thinly myelinated (A- $\delta$ fibers), or unmyelinated (C fibers) [37].

A bipolar neuron has two processes extending directly from the cell body, one forming the axon end, and the other (opposite side) forming the dendrite tree. This type of cell looks simply like a chain, and one end can be encapsulated to become a sensor and the other end synapses the cell body of another neuron. A bipolar neuron of this type has its cell body along the axon (see Figure 1). The peripheral A- $\delta$ afferent fibers that have nociceptors and thermoreceptors have diameters in the range $1-6$ micro-meters. The thermoreceptors are of two types-the cold thermoreceptors respond to the range $\left(5^{\circ}-40^{\circ} \mathrm{C}\right)$ whereas the warm thermoreceptors fire signals when the temperature is within the range $\left(29^{\circ}-45^{\circ} \mathrm{C}\right)$ [38]. We refer to Figure II.11 of [35] for the skin structure down to the subcutaneous layer. The sensory nerves with branches can be ending in 1) free endings which detect heat and pain; 2) Pacinian corpuscle sensing pressure; 3) Meissner's corpuscle which senses touch; 4) Merkel disc also senses touch. Some elastic fibers are thinly populated. The curvy structures represent the sweat glands, which are innervated by sympathetic nerves only. The representative hairs with their papilla of hair follicles and the sebaceous glands are also shown there. The three layers of the skin are well-known: epidermis, dermis, subcutaneous, and are labelled. Sensors 2)-4) encapsulate the nerves at the axons, and the free endings are simply un-capsulated axon terminals [39].

Notice that the cold receptors are supplied by both A- $\delta$ or C fibers, but the warm receptors, which are relevant to warm Bian stone treatment applied to the skin, are only supplied by the C fibers. Cranial nerves (I, V, VII, IX, X), which are parasympathetic nerves, are relevant to our discussion in this paper. These cranial fibers have diameters ranging from around 0.8 to $10 \mathrm{~mm}$ [40]. The 


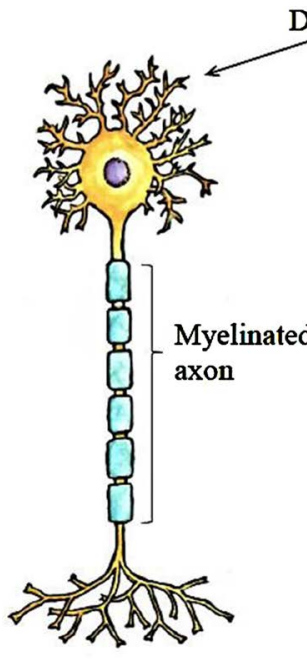

(a) Motor neuron (multipolar neuron)
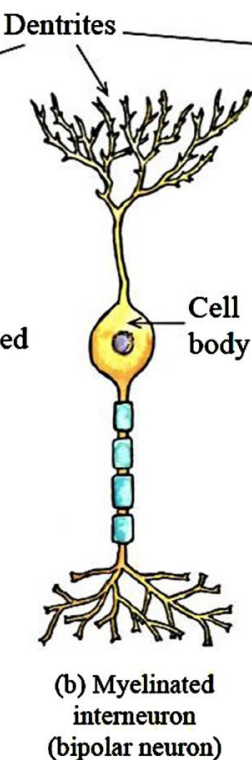

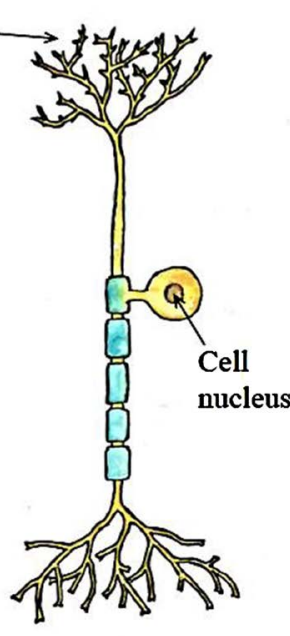

(c) Sensory neuron (pseudo-unipolar neuron)

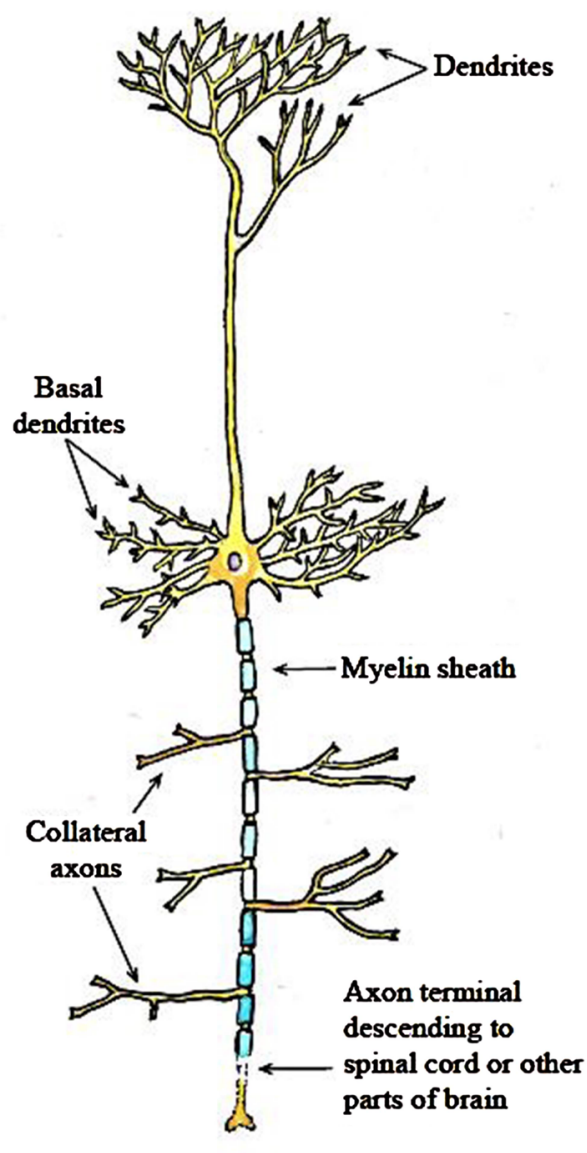

(d) Pyramidal cell in neo-cortex

Figure 1. Four types of neurons discussed in this paper. (a) Multipolar neuron [35]. (b) Bipolar neuron [36]. (c) Pseudo-unipolar neuron Figure 7 of [37]. (d) Pyramidal neuron [42].

myelination specification of different neurons is indicated in Figures 1(a)-(d). Note that the bipolar neurons in the peripheral are not myelinated, whereas those in the neo-cortex are, for reason to be clear when we come to Section (5.3). We would emphasize that using the rat models, high density of nerve endings was found at many acupoint sites [41].

In Figure 1(d) we show schematically a pyramidal neuron which has many dendrites growing from the cell body. One dendrite, however, extends to a longer distance and branches off to a dendrite tree. The axon is usually long, and the main axon terminal targets the spinal cord, thalamus, or other parts of the brain. Along the myelinated axon, there are other axons, called collateral axons, extending along sideways, providing the ability to synapse the cell bodies of other neurons in the brain, especially those in the cortex region. Pyramidal neurons have their cell bodies in the neo-cortex (within $\sim 2 \mathrm{~mm}$ on the "outer part" of the cortex) of the brain. The axon branch can have some portions myelinated whereas the other portions "naked" [42].

Apart from the four types of neurons, there are many more neuron types, of the order of tens or hundreds in the brain, but we will not discuss them in this paper. 


\subsection{Two Basic Neurotransmission Processes}

Schematic representation of common neurotransmission and retrograde neurotransmission processes are shown in Figure 2. Figure 2(a) indicates that the

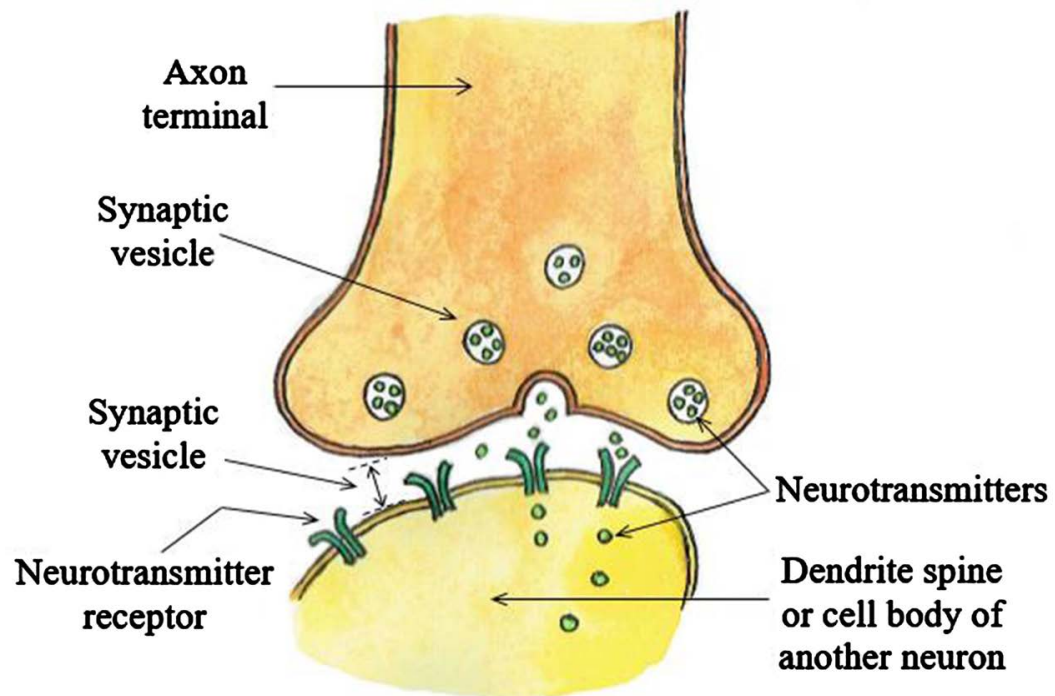

(a)

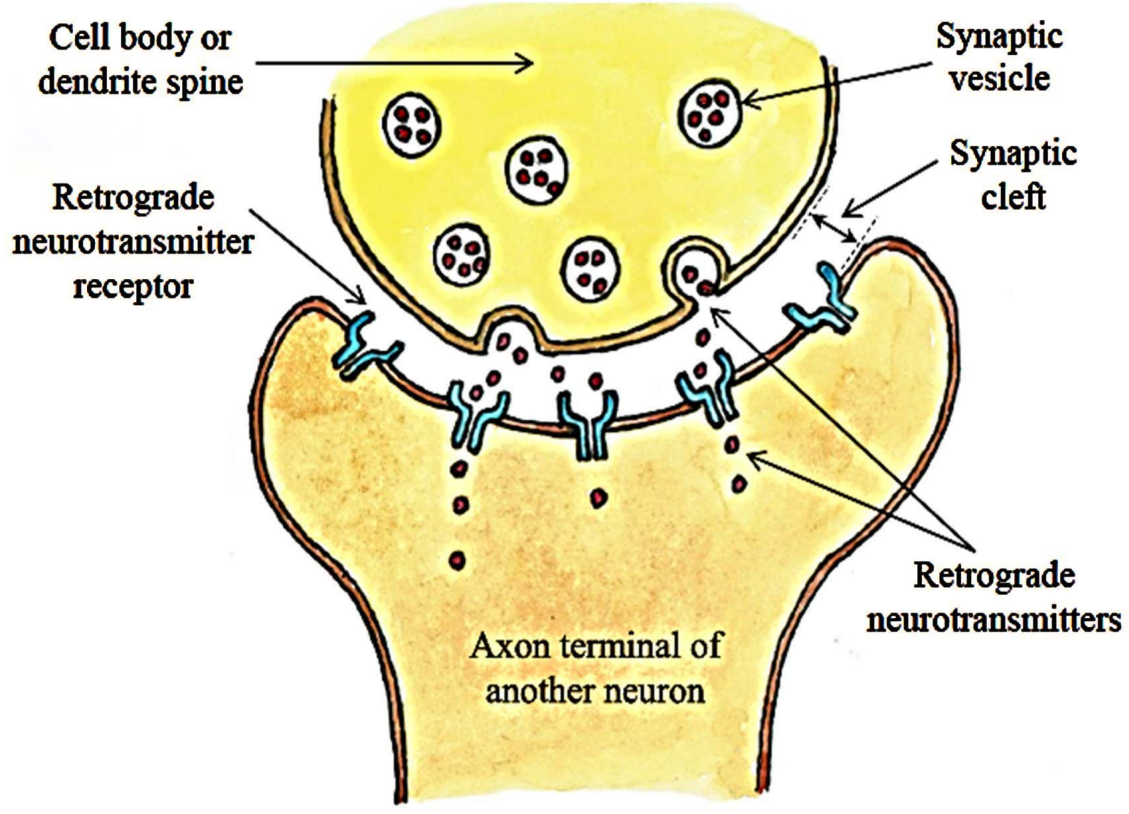

(b)

Figure 2. Schematic representation of common neuro transmission and retrograde neurotransmission. (a) An axon of a neuron transmits neural transmitters (green small circles, such as GABA, being kept in the vesicle) to the dendritic "tip", the dendrite spine, or the cell body of another target neuron. The excretion process is exocytosis. The targeted cell has the special neurotransmitter receptor (deeper green). (b) Transmission between neurons can go the other way. The cell body and dendrite spines also have vesicles which keep special "retrograde neurotransmitters" (red small circles). Through exocytosis, these retrograde neurotransmitters can be expelled, and are received by the special retrograde receptors (blue) at the cell body or axon of another neuron. 
neurotransmitters (small green circles) are kept in synaptic vesicles at the axon terminal. An axon of a neuron can transmit these neurotransmitters (such as gamma-aminobutyric acid or GABA) to the dendritic "tip", called dendrite spine, or the cell body of another target neuron via simple exocytosis. The targeted cell has the special neurotransmitter receptors (deep green with Y-shape structure) for accepting these transmitters when they pass through the synaptic cleft. The signal is then passed on to the axon terminal of the second neuron. Figure 2(b) indicates the "retrograde transmission" from the cell body and dendrite spines which also have vesicles storing the special "retrograde neurotransmitters" (red small circles, such as nitric oxide) [43]. Through exocytosis, these retrograde neurotransmitters can be expelled, passing though the synaptic cleft, and are received by the special retrograde receptors (blue) at the axon terminal of the target neuron.

\subsection{The Balance of the Sympathetic and Parasympathetic Nervous Systems via Various Means, Including Stimulating the Meridians}

The autonomic nervous system functions to regulate the body's actions. The sympathetic nervous system (SNS) triggers the readiness/action of the body to take on a fight-or-flight response. It is, however, constantly active at a basic level to maintain homeostasis of a large number of organs [44]. Therefore, the SNS is called "antagonistic", accompanied with increased metabolic rates of the innervated organs, leading to increase of the heart rate, increase of breathing rate, dilation of the pupils in order to be aware of the changes in environment, constricting blood vessels, contraction of the vesical and rectal sphincters, together with inhibition of peristalsis and alimentary secretion, so that the digestive system and energy storage processes are stopped, and that more oxygenated blood is maintained in the skeletal muscles, heart, lungs and brain. To achieve the above purposes, the SNS contains nerve fibers which are mainly adrenergic.

On the other hand, when threats/pressures are relieved, the body should work on a system of organs leading to energy storage in general; this action includes the parasympathetic system (PNS) that contains chiefly cholinergic fibers, so that a range of secretions are induced, to contract the smooth muscles of the alimentary system [45]. The consequence is that more food is digested and energy stored, accompanied with a low heart rate. Apart from the diaphragm (which is only innervated by SNS neurons), all organs are innervated by both SNS and PNS nerves, so that a balance can be reached under a wide range of conditions, for survival. Generally, when the SNS of an organ is increased, the PNS of the same organ is decreased, and vice versa. There is only one exception: the genital organ requires the activation of both nervous systems during sexual action. Many disorders are caused by imbalance of the SNS and PNS. In TCM, attempts are made to balance these two systems by specific maneuver of the nerves involved, the action of which is dependent on the type of disorder 
involved.

\section{Basic Operations of Respiratory Action}

The respiratory system includes organs as briefly introduced in Section (1.2). As details of various parts will be discussed later, we show only the simple schematic breathing action in Figure 3. Normally, bronchi are in a somewhat "collapsed state", with a star-shaped-like lumen. This means that the lumen cells and the mucociliary apparatus has a chance to be in contact with most of the (external) materials present in the lumen and, if necessary, secrets mucus to move the materials upwards and out of the system by action like coughing. Two sets of nerve, called anterior pulmonary plexus and posterior pulmonary plexus respectively, innervate the lungs at the anterior and posterior roots. Of course, they are accompanied by the associated blood vessels and lymphatic vessels into the bronchi and lungs. Each pulmonary plexus contains sympathetic nerves from ganglions of the sympathetic trunk and parasympathetic nerves from the vagus nerves descending from the brain stem. We will show later that the cervical spinal nerves down to thoracic spinal nerve T1 must form a group (the cardiac-pulmonary plexus to innervate the heart and the lungs) because these two organs must operate in harmony to supply oxygen and nutrients to the muscles if a skeletal action is to take place.

In general, postganglionic sympathetic nerve fibers from the sympathetic trunks are bronchodilators, vasoconstrictors, and inhibit glandular secretion. Parasympathetic nerve fibers from the vagus nerves are bronchoconstrictor, vasodilator, and secretomotor to the glands.

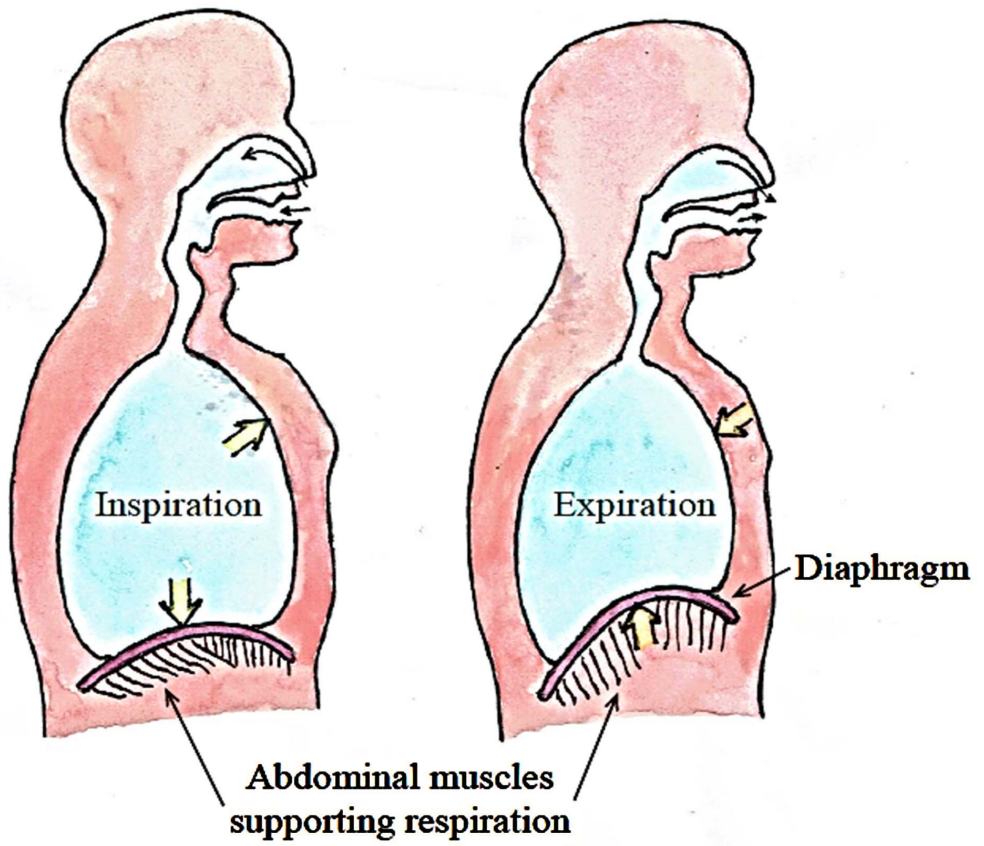

Figure 3. A self-explanatory simple diagram representing the action of inspiration and expiration. 


\section{Mechanical Stimulation of the Lung Meridian Can Activate Sympathetic Nerves and Improve Respiratory Function}

\subsection{Biomedical Consequences as the Lung Meridian Is Stimulated Mechanically-Starting with Three Nerves Which Branch off and Enter the Cervical Spinals C5 to C8, T1}

On the left of Figure 4, locations of the 11 known acupoints LU1 to LU11 are marked. Along the forearm and arm, the main nerves are the Ulna nerve, Medium nerve, the Radial nerve, \& the Musculocutaneous nerve (refer to Fig.29.2 of [46]). These four main nerves, together with some smaller cutaneous branches joining up in a complicated manner to form the Medial chord, the Lateral chord, and the Posterior chord as shown on the right side of Figure 4. LU11 (shaoshang) is near the end of the Medium nerve of the thumb. LU10 (yuji), 9 (taiyuan), 8 (jingqu), 7 (leique) are also close to the Medium nerve of the wrist region. LU6 (kongzui) is close to the lateral ante-brachial cutaneous nerve, which is a branch of the Radial nerve. LU5 (chize) is close to the main Radial nerve at the brachioradialis. LU4 (xiabai) is near the Musculocutaneous or the lateral brachial cutaneous nerve. LU3 (tianfu) is at the Musculocutaneous nerve of the upper bicep brachii, and LU2 (yunmen) is below the supraclavicular and near the posterior chord which is built from 4 nerves joining the spinal segments C5, C6, C7, C8, and T1 [47]. LU1 is near the middle of the supraclavicular and the posterior chord too. On the right side of Figure 4, we see that several nerves, such as the Ulna nerve is composed of 2 nerves entering both the T1 and C8 spinal segments. Other nerve roots have more branches enclosed as shown. Suppose an acupoint (the LU11) along the Lung Meridian is excited by either manual acupuncture, acupressure or moxibustion. There are different nerve sensors at the site of application. The Pacinian corpuscles (responding to pressure), Ruffini endings (also responding to pressure) and free nerve endings (responding to heat and pain) are excited. The signals pass through several spinal nerves to enter the spinal cords $\mathrm{C} 5,6,7,8$, and $\mathrm{T} 1$, as clearly shown in the above figure. Therefore, stimulation by acupuncture or acupressure at the acupoints of the Lung Meridian will activate the nerve fibers entering T1 up to C5 spinal segments in this diagram. Note that spinal nerve $\mathrm{C} 5$ has a branch going up to join $\mathrm{C} 4$, which has sub-branches innervating $\mathrm{C} 4, \mathrm{C} 3$ and above. There are many effects in responding to the stimulation along the Lung Meridian. Before we follow the signal through $\mathrm{C} 5$ to $\mathrm{C} 4$, we need to understand some basic structure of the cross-section of a spinal cord in the next section.

\subsection{Introduction to the Connection of the Spinal Nerves and the Spinal Cord}

Referring to Figure 5, the grey matter has a "butterfly" shape, which varies in shape in different spinal cords. The two "extension" to the back are the dorsal horns, and the two on the "front" are ventral horns. This greyish structure is full of neuron cell bodies, cell processes including axons and dendrites, glial cells 


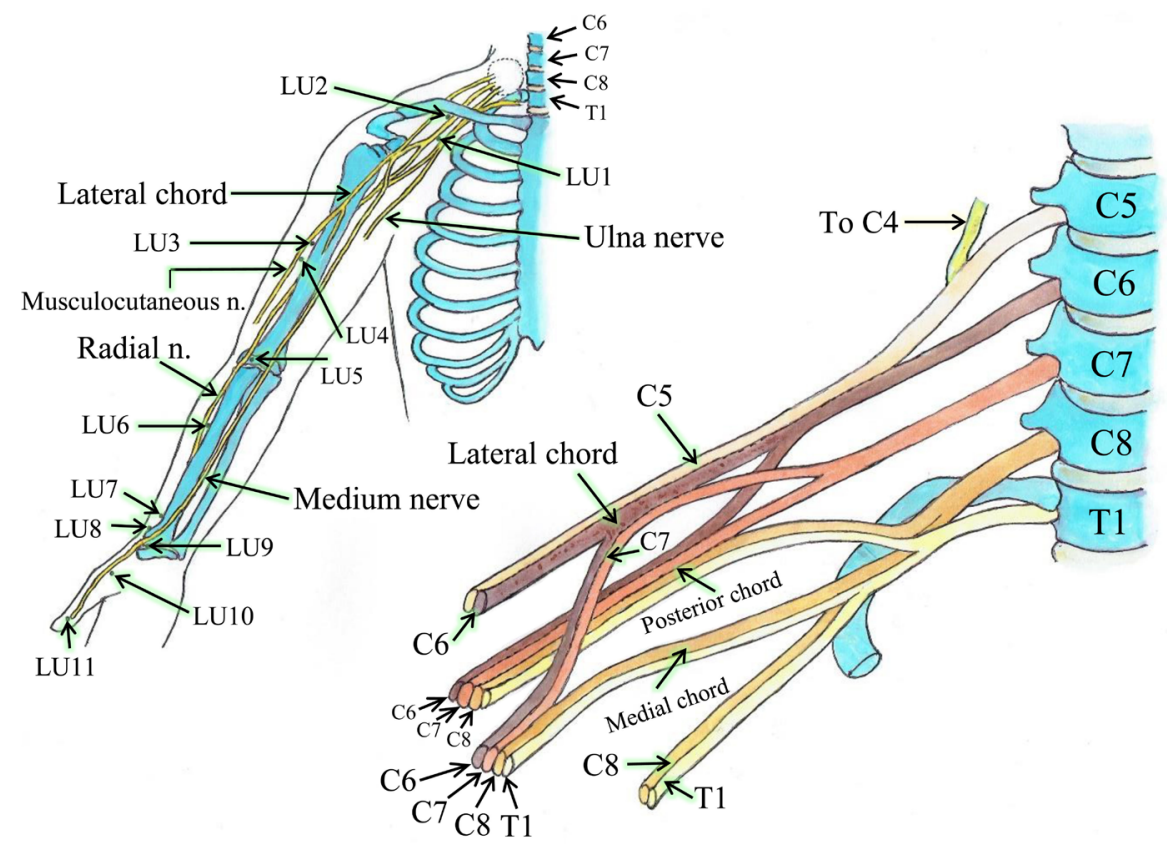

(a)

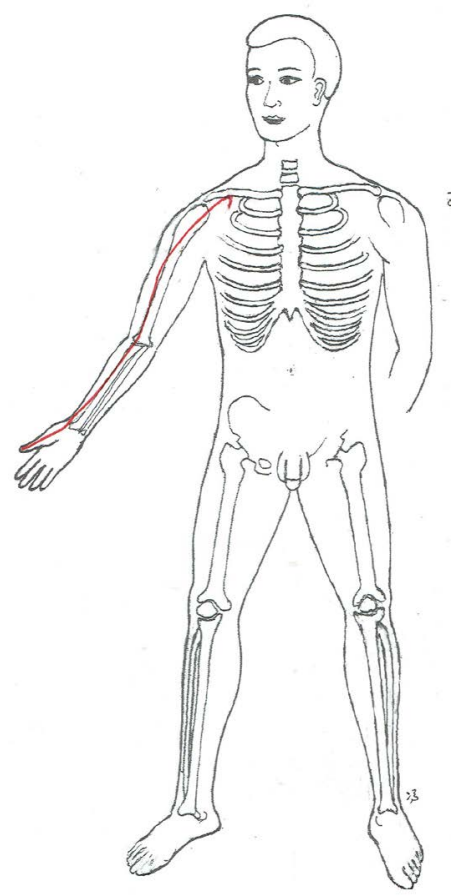

(b)

Figure 4. (a). On the left, locations of the 11 known acupoints LU1 to LU11 of the Lung Meridian are marked. Along the forearm and arm, the main nerves are the Ulna nerve, Medium nerve, the Radial nerve, and the Musculocutaneous nerve. These four main nerves, together with some smaller cutaneous branches joining up in a complicated manner to form the Medial chord, the Lateral chord, and the Posterior chord with themselves or branches joining $\mathrm{C} 5$ to $\mathrm{T} 1$. The spinal nerve at $\mathrm{C} 5$ has a branch going up to join $\mathrm{C} 4, \mathrm{C} 3, \mathrm{C} 2$, \& C1. The part on the nerve roots entering spinal cords C5, C6, C7, C8, and T1 on the right side is a hand-painted adaptation of Figure 19 of [46], adding different colors. (b) The Lung Meridian as revealed on the skin of a body model (compare p. 73 of [47]). 


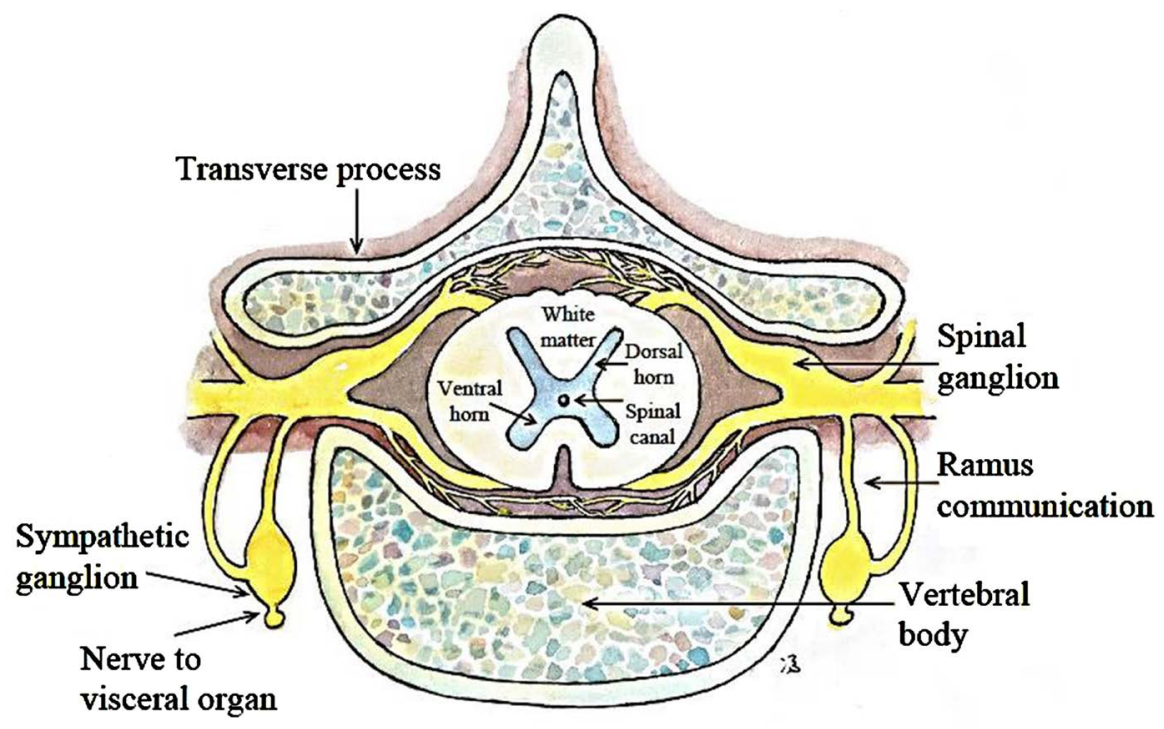

\section{Ventral view}

Figure 5. The cross-section of a typical cervical spinal cord. The "butterfly" shaped structure is the grey matter, and the whitish part "surrounding" the grey matter is the white matter. The cerebrospinal fluid flows through the little circle in the middle between the brain ventricles and the sacrum segments. Viewing down, we observe two yellow spinal nerves (from the brachial plexus, related to the Lung Meridian outside this diagram) entering the space between the bony vertebral body and the bony transverse processes. These four branches of the spinal nerves join the two ventral horns and two dorsal horns of the grey matter, after passing through certain parts of the white matter. There are nerves branching from each spinal nerve, each of which leads to a smaller ganglion near an organ, like the root of a lung [48] [49].

(which play only passive roles) and blood vessels (mainly capillaries) for nourishing the neurons. There are of course collagen layers embedding each neuron and blood vessel. The white matter also contains similar living constituents as the grey matter, but the neurons in the white matter form mainly many "longitudinal tracts" joining various parts of the whole spinal cord, and the brain. In short, in the grey matter, many specific signals are transmitted between interneurons and other neurons (motor and sensory neurons); there is also interchange of signals among the interneurons themselves. There are numerous signals being transmitted between neurons in the grey and white matters also (Fig. 11.1 of [35]).

Note also that we observe two yellow spinal nerves (originated from the brachial plexus) entering the space between the bony vertebral body (on the ventral side) and the bony transverse processes at the back. These four branches of the spinal nerves, after passing through certain parts of the white matter, join the two ventral horns and two dorsal horns of the grey matter. There are nerves branching to the outside (the bony) region from each spinal nerve. On the ventral side, a side branch nerve leads to a sympathetic ganglion (some detailed structure will be described in later Sections), which has also branching nerves, one of which leads to a smaller ganglion near an organ. On the dorsal side, there 
are also ganglions close to the transverse processes of the vertebra bone segments. Note that four spinal nerves (C1-C4) are joined to the top/superior sympathetic ganglion, two spinal nerves $(\mathrm{C} 5, \mathrm{C} 6)$ are joined to the middle sympathetic ganglion, whereas three spinal nerves $(\mathrm{C} 7, \mathrm{C} 8, \mathrm{~T} 1)$ are interacting inside the inferior or stellate sympathetic ganglion. The spinal canal is a small tube through which the cerebrospinal fluid flows [3]. Now we can follow up our analysis stated at the end of Section (4.1).

\subsection{Stimulating the Lung Meridian Starts off the Respiratory Action by Activating the Diaphragm via the Phrenic Nerve and Activating the Parasympathetic Nerve via Dragging the Connective Tissues Connecting the Vagus Nerve around the Esophagus Hiatus Indirectly}

Refer to Figure 6, before entering the $\mathrm{C} 5$ segment, a side branch of the $\mathrm{C} 5$ nerve goes up to become the $\mathrm{C} 4, \mathrm{C} 3$ and $\mathrm{C} 2$ spinal nerves in a rather intermingled manner. The spinal $\mathrm{C} 4$ nerve as the main sympathetic nerve, together with branches of the $\mathrm{C} 5$ and $\mathrm{C} 3$ nerves form the bilateral phrenic nerves. The phrenic nerves passing "edge on" between the heart and the lungs, with some side branches innervating the heart, joining the diaphragm organ (refer to Fig. 130.1 of [46] and Fig. II.16 of [35]). This pair of nerves, causing the movement of the diaphragm to move in a cyclic manner, are "initiators" of the breathing function. Note that the diaphragm is a very particular organ, separating the upper abdomen, where the fight or flight readiness is executed, and lower abdomen where energy is being stored and wastes deposed off. There is only sympathetic nerve innervating this organ. As we follow the action of activating certain acupoints of the Lung Meridian, signals are sent by a rather wide range of spinal nerves, entering $\mathrm{C} 5$ to $\mathrm{T} 1$ in Figure 4. Therefore, stimulating acupoints at the nerve fiber connecting C5 (which has connection to C4) would enhance diaphragm movement. It is interesting to note that the vagus nerve, after descending from the brain stem, will pass through the hiatus of the diaphragm as shown in Figure 6 . Thus, as one breathes, particularly deeply, the vagus nerve tends to be "dragged" in up-down periodic motion. This might be the reason for the observed fact that deep breathing exercise like yoga and certain meditation might cause relaxation. The spinal nerve $\mathrm{C} 3$ branches off to $\mathrm{C} 2$ and $\mathrm{C} 1$ also. $\mathrm{C} 1$ is associated with vision and we leave that alone here. In physiological term, the initiation of breathing has already begun. Air would flow in through the nose.

\subsection{Stimulating the Lung Meridian Affects the Vessel Dilation and Mucosal Secretion of the Nasal Cavity, Leading to Relief of Nasal Congestion}

Nerves innervating the nose cavity, nasal glands for detecting smell (see Figure 6 of [3] and Fig. II.100 of [35] on the structure of the olfactory nerve), for vessel dilation and mucosal secretion. Whereas cranial nerve (CN) I forms the olfactory nerve, two parasympathetic cranial nerves $(\mathrm{CN}$ V/trigeminal nerve and $\mathrm{CN}$ 


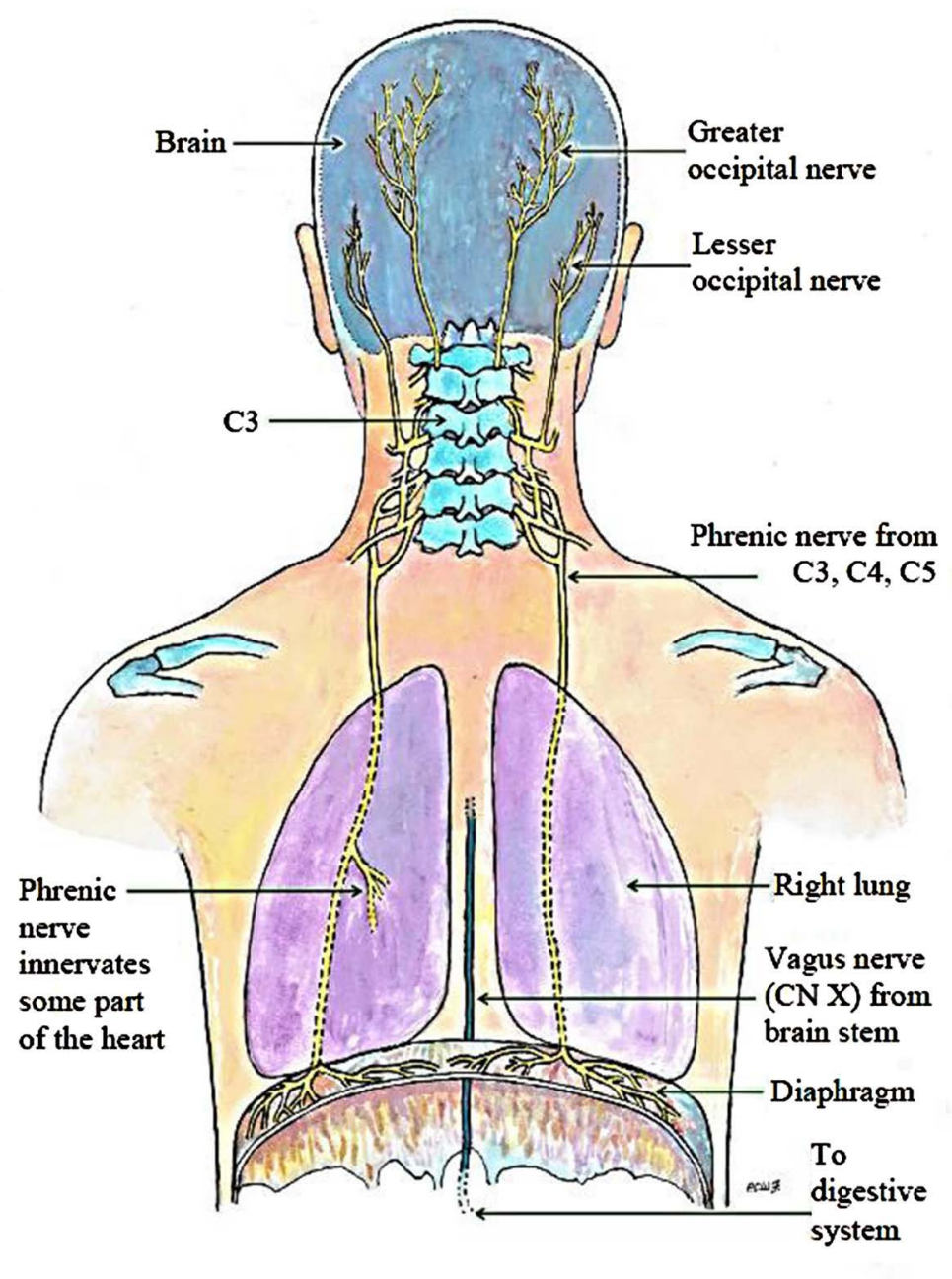

Posterior view

Figure 6. Activation of the Lung meridian would stimulate the Medium, Radial, the Musculocutaneous nerves which have branches entering the white matter and grey matter of the spinal cords C5, 6, 7, 8, T1 as explained. The lateral musculocutaneous nerve branches from the C5 spinal nerve goes up to upper cervical nerves with a little intermingled structure. The main spinal nerve innervating the diaphragm is the $\mathrm{C} 4$ nerve (see Fig. 130.1 of [46] and Fig. II.16 of [35]). The vagus nerve passes through the hiatus to innervate the digestive system.

$\mathrm{VII} /$ facial nerve) participate in homeostasis of the mucosal secretion of the nose cavity and toning the blood vessels of the nose. The smell detected is fed to the dorsal respiratory group of the brain stem to affect the depth of breathing (see input to the dorsal respiratory group discussed in Section (5.6)). The CN V nerve, after passing through the ethmoid foramina, is called the anterior ethmoidal nerve which enters the upper part of the nose cavity, as shown on the left upper corner of Figure 7. Another parasympathetic nerve, i.e. CN VII emerges from the inferior part of the pons. Neuron from the superior salivary nucleus synapses the second neuron in the geniculate ganglion; this neuron becomes part of the vidian nerve and synapses the third neuron in the pterygopalatine ganglion. This third neuron innervates the mucosal glands in the nose. A 


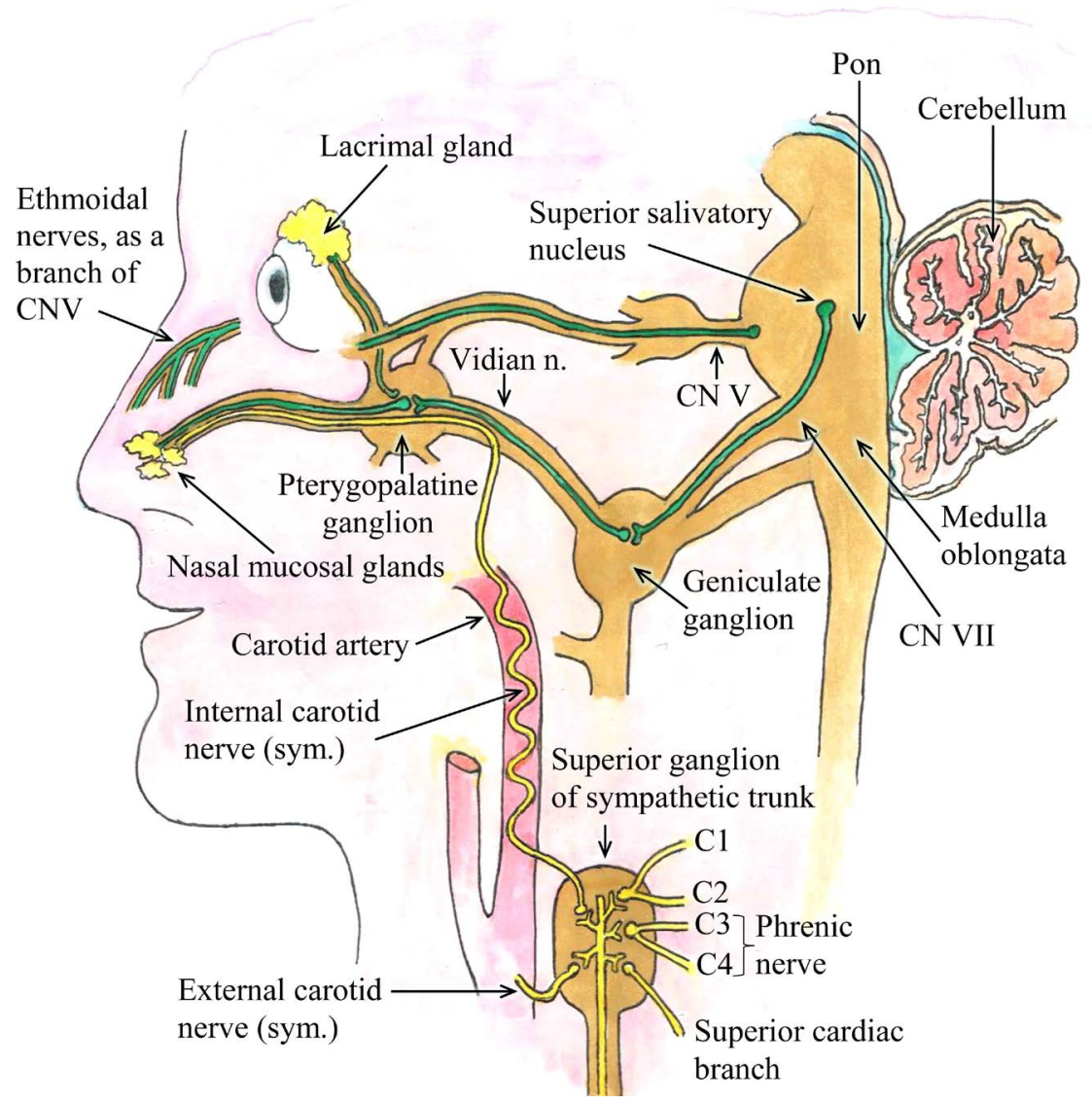

Figure 7. Two parasympathetic cranial nerves (CN V/trigeminal nerve and CN VII/facial nerve) participate in homeostasis of the mucosal secretion of the nose cavity and toning the blood vessels dilation of the nose. The CN V nerve, after passing through the ethmoid foramina, becomes the anterior ethmoidal nerve to enter the upper part of the nose cavity. CN VII emerges from the inferior part of the pons. Neuron from the superior salivary nucleus synapses the second neuron in the geniculate ganglion; this neuron becomes the vidian nerve and synapses the third neuron in the pterygopalatine ganglion. This third neuron innervates the mucosal glands in the nose. A neuron from the pterygopalatine ganglion also innervates the lacrimal gland of the eye. The axons of neurons of the sympathetic truck synapse four spinal nerves (C1-4, yellow) plus the superior cardiac branch (yellow). The internal carotid nerve forms a network-like structure in the internal connective tissue layer of the carotid artery. This nerve is the main sympathetic nasal nerve to tone nasal glands. The CN I which forms the olfactory nerve is not shown.

neuron from the pterygopalatine ganglion also innervates the lacrimal gland of the eye. The superior sympathetic ganglion (orange) is the first sympathetic ganglion of the sympathetic trunk [49]. The axons of neurons of the superior sympathetic ganglion (orange) of the sympathetic trunk synapse four spinal nerves (C1-4, yellow) plus the superior cardiac branch (yellow) of the sympathetic nerve. As shown in Figure 7, one branch coming out of the superior sympathetic ganglion is the internal carotid nerve which forms a network-like structure (represented by a wavy structure for simplicity) in the internal connective tissue layer of the carotid artery. This nerve is the main sympathetic nasal nerve to tone the action of the nasal glands. Both sympathetic and parasympathetic 
nerves innervate the "entry organ", i.e. the nose of the respiratory system, as expected (see also [50]). About half of the obstruction of air flow occurs in the nose and is a risk factor for sleep-disordered breathing [51]. Therefore, it is important that both the sympathetic and parasympathetic nerves supplying the nose should work in harmony. The microvasculature of the nose consists of three parts: 1) A subepithelial network of capillaries, providing nutrients to the epithelium and glands; this system also allows passage of water into the lumen for evaporation and air-conditioning. 2) A system of capacitance vessels or sinuses. When these vessels distend, the nasal lumen is blocked, allowing very small amount of air to pass through. When they are constricted, the nasal passages are then open. The amount of constriction affects the filtering and air-conditioning functions of the nose, as well as the amount of air allowed to pass through. 3) Small arteries, arterioles, and arteriovenous constitute the system of resistance vessels, allowing rapid passage of blood through the mucosa. Sympathetic nerve stimulation causes constriction of the resistance vessels by the alpha-adrenergic mechanism and the venous vessels serve as capacitance vessels [52]. Parasympathetic nerve stimulation causes non-cholinergic dilatation of both resistance and capacitance vessels. It has been known long ago that the nasal mucosa is drained by two separate venous systems mentioned in 2) and 3). Note that the nasal mucosa receives tone discharges from the sympathetic nerves but not from the parasympathetic nerves. Hence, nasal congestion could be due to a withdrawal of sympathetic discharge than to an overactivity of the parasympathetic nerves. Bridging the east and west, thus stimulating the Lung Meridian could lead to relief of nasal congestion [53].

\subsection{Stimulating the Lung Meridian Would Send Signals to the Larynx-Pharynx-Trachea System}

Note that the inferior ganglion of the vagus nerve has connection with the superior ganglion of the sympathetic trunk. The left side of Figure 8 shows the internal laryngeal (sensory, dotted yellow lines) and external laryngeal (motor, solid yellow lines) sympathetic nerves which exit from the superior ganglion of the sympathetic trunk [54].

The external laryngeal nerves innervate the laryngeal constrictor muscles, causing the esophagus to open and close during respiration, in opposite phase to the cyclic action of the vocal cords [55].

Note that the cranial nerve IX (glossopharyngeal nerve), carrying both sensory and motor neurons, exit the brain stem from the upper medulla, at a site very close to where CN X exits. CN IX leaves the skull through the jugular foramen. It descends and innervates the palatine tonsil, the mucous membrane of the fauces (the arched opening at the back of the mouth leading to the pharynx) and the base of the tongue. Thus the pharynx is innervated by a mixture of the CN IX and $\mathrm{CN}$ X nerves. The combined system has motor neurons (to open and close the vocal cords and swallowing action [55]) and sensory neurons (for sense of taste of the posterior of the tongue-not shown here). For simplicity, we present 
the bluish green nerves to represent such a mixture nerve system [56] [57] on the right side of Figure 8. Viewing down the glottis, with the vocal cords open, the observer will see rings of the trachea. Below the vocal folds, the larynx is mainly innervated by the recurrent laryngeal nerves.

During breathing, if the vocal cords are not open, the person is in a dangerous situation. Stimulating the Lung Meridian participate in toning the operation of the larynx and the pharynx.

\subsection{Stimulating the Lung Meridian Would Activate the Scalene Muscles for Inspiration and Cause Bronco-Dilation via the Pulmonary Plexuses}

The electromyograms of the scalene, sternocleidomastoid, and trapezius muscles of 40 severe COPD patients were measured during resting breathing in 1) seating posture; 2) supine posture [58]. It was found that all patients demonstrated strong inspiratory contraction of the scalene muscles in 1). Only $10 \%$ of the patients showed some inspiratory activity in the sternocleidomastoid and no
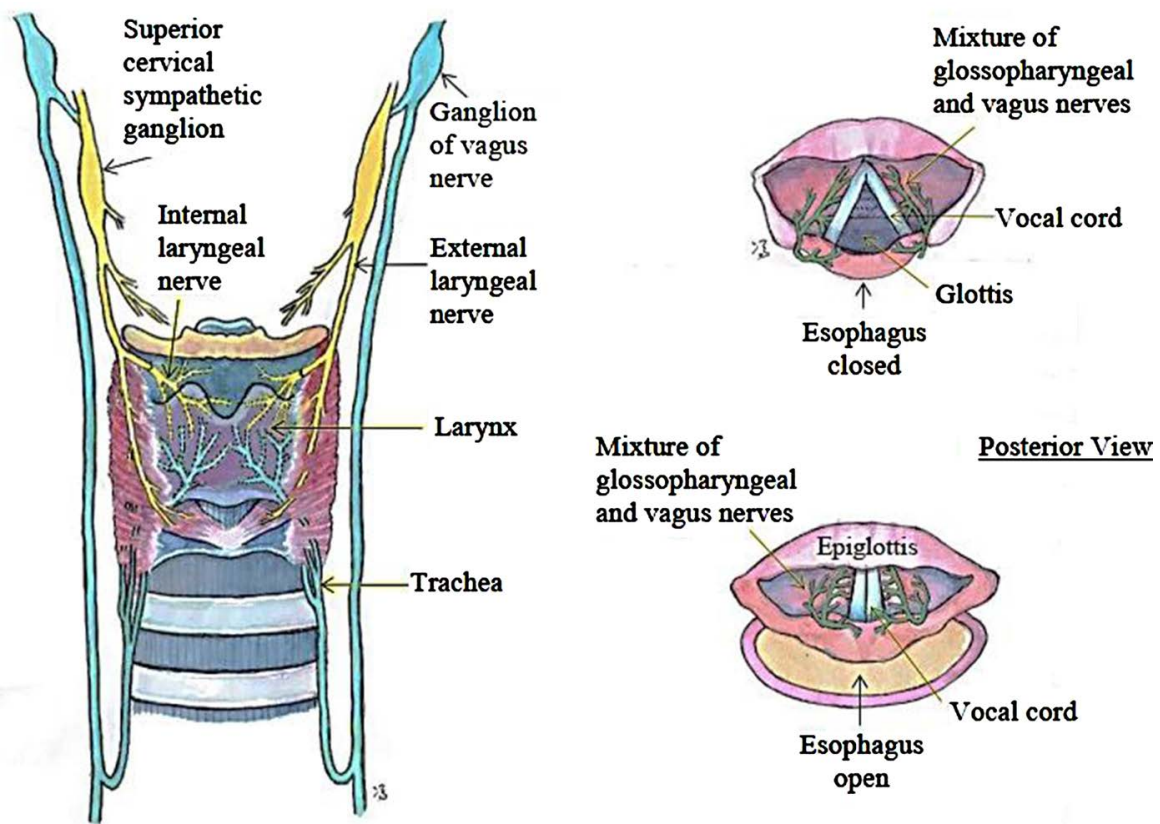

Figure 8. The left side shows the sympathetic nerves from the superior ganglion of the sympathetic trunk form the internal (dotted yellow lines) and external laryngeal (solid yellow lines) nerves which innervate the larynx. These nerves contain some portion of the vagus nerves. A branch of the vagus nerve exits from each inferior parasympathetic ganglion, descending to a site near the aortic arch and turns up to form the recurrent laryngeal nerve (blue) on each side, innervating the larynx also [54]. On the right side, we indicate the simplified pharynx system. Viewing down the glottis, with the vocal cords open, the observer will see rings of the trachea. The pharynx is innervated by a mixture of the CN IX and CN X nerves, which exit the brain stem at proximity. The combined system has motor neurons (to open and close the vocal cords and swallowing action) and sensory neurons (for sense of taste of the posterior of the tongue-not shown here) [55]. For simplicity, we present the bluish green nerves to represent such a mixture nerve system. 
activity in the trapezius muscles. In posture 2), only scalene muscles were involved in inspiration. Remark that the scalene muscles are innervated by the $\mathrm{C} 4$, C5, C6 spinal nerves [59]. This aspect will be amplified in another paper on the Lung Sinew Channel shortly later.

On the other hand, based on animal models, it was established long ago that the lungs are innervated primarily from the anterior and posterior pulmonary plexi, constituting sympathetic nerves and the vagus nerves [60] [61] [62]. In general, the sympathetic nerves cause bronchodilation and blood vessel constriction, whereas the vagus nerves constrict the bronco-vessels but dilate the blood vessels.

Using the technique of retrograde axonal tracing combined with immunohistochemistry (applied to the rat model), and based on analysis of the amounts of calcitonin gene-related peptide (CGRP), there was evidence showing that effectively the vagus nerves are more involved in the relaxation of the trachea. Note that CGRP is synthesized and released from sensory nerves in the central nervous system can be released to the peripheral. It can function as a bronco-dilator [63].

The sensory innervation of the lungs was shown to originate mainly from the ipsilateral dorsal root ganglia (45\% of cells CGRP-immunoreactive) at levels $\mathrm{T}_{1}-\mathrm{T}_{6}$, implying there was much stronger influence of the sympathetic nerves at these spinal levels in causing bronchodilation of the rat's lungs themselves [64]. Later, there was evidence in guinea-pig model study that T1-T4 spinal nerves are engaged more significantly in sympathetic motivation of the lungs directly (see e.g. [65]). In view of the above evidence, we consider that the spinal nerves $C 7$, C8, T1 constitute the major part of the cardiac-pulmonary plexus of humans, and surely stimulating the T1 spinal nerve can activate the bronco-dilation effect in the lungs and activate the heart function (see Fig. 11.38 of [66] [67]). On the upper left of Figure 9, the afferent sensory neurons (yellow) from the brachial plexus (see Figure 4 also), painted in green, enters the spinal cord T1. The circular shaped small body indicates the cell body of the neuron, which is rather long in the limbs. The sensory neuron has its dendrite inside the grey matter. The said dendrite synapses an inter-neuron (brown) which is short and within the "butterfly". The inter-neuron passes on the message to another neuron (orange) which stretches through the white ramus (a nerve tube in green color, schematically) to the stellate/inferior cervical ganglion (circular shape in green), which is a "station' where neurons exchange signals (or synapses). After synapsing, the motor neuron (red) coming out of the stellate ganglion is contained in a nerve tube (green). This neuron enters another circular shaped junction (prevertebral ganglion) and passes its message to a region called "pulmonary plexus". On the other hand, the vagus nerve ( $\mathrm{CN} \mathrm{X}$, blue) descends from the nodose ganglion near the brain stem and enters the pulmonary plexus also. The pair of sympathetic and parasympathetic nerves from the pulmonary plexus enters the root of one lung to innervate the pulmonary vasculature. The $\mathrm{C} 7, \mathrm{C} 8$ nerves in the stellate ganglion are not shown for simplicity. 


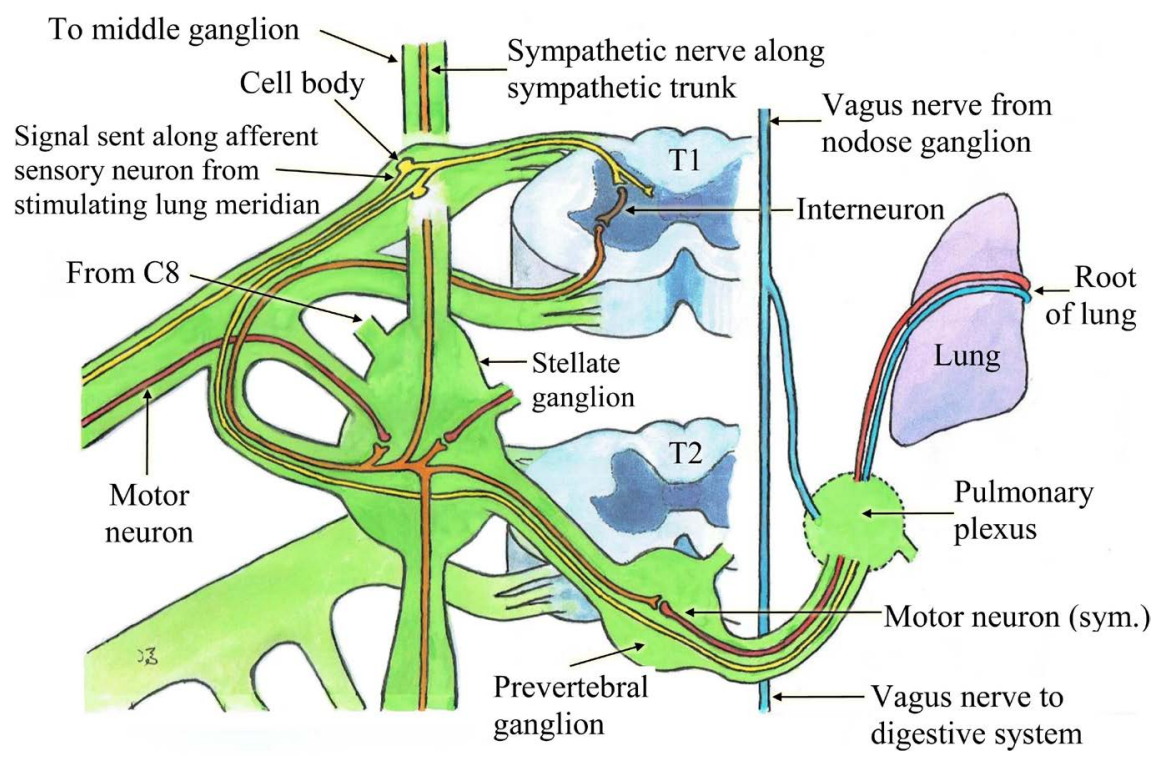

Figure 9. Afferent sensory neurons (yellow) enclosed by a nerve fiber from the upper limb (referring to Figure 4), enters the spinal cord T1. The sensory neuron has its dendrite inside the grey matter. The said dendrite synapses an inter-neuron (brown). The inter-neuron passes on the message to the middle part of the stellate cervical ganglion (green). The neurons coming out of the stellate ganglion are contained in a nerve tube (green). This nerve tube enters the prevertebral ganglion. A (red) motor neuron joins the pulmonary plexus. The vagus nerve ( $\mathrm{CN} \mathrm{X}$, blue) descends from the nodose ganglion near the brain stem and enters the pulmonary plexus also. This pair of nerves from the plexus enters the root of one lung [67]. The motor neuron from C6 (outside the diagram) innervate the scalene muscles to trigger inspiration, but not shown in this diagram. We only present the $\mathrm{T} 1$ nerve to represent the cardiac-pulmonary nerve plexus, which includes $\mathrm{C} 7, \mathrm{C} 8$ also in the stellate ganglion.

\section{Stimulate the Acupoint Lung 11 and Follow the Signal All the Way to the Somatosensory Cortex of the Brain}

\subsection{Introducing the Spinothalamic, Anterior Corticospinal, Lateral Corticospinal Tracts Which Are Relevant to Stimulating Acupoints of the Lung Meridian in Signal Transmission and Reception}

First, let us describe some basics of the grey and white matter of the spinal cord again. From experience for centuries, we can in general consider that needle acupuncture with twirling technique of stimulation would cause the feeling of dull pain, and moxibustion leads to feeling of hyperthermia.

The cranial nerve I pass through lamina I (light blue in Figure 10, see any text on neuroanatomy) along the cord all the way down. Note that inside the spinal cord, the vagus nerve passes down lamina $\mathrm{X}$ (blue) around the small spinal tube (yellow) at about the center of the cord. The white matter of the spinal cord (white in Figure 10) is full of ascending and descending neurons from certain spinal cords up the brain and descending neurons from the brain down the cord. For this paper, we are interested in the sensory neurons passing the ascending "spinothalamic tract" through some of spinal cords (yellow-orange) along directions 


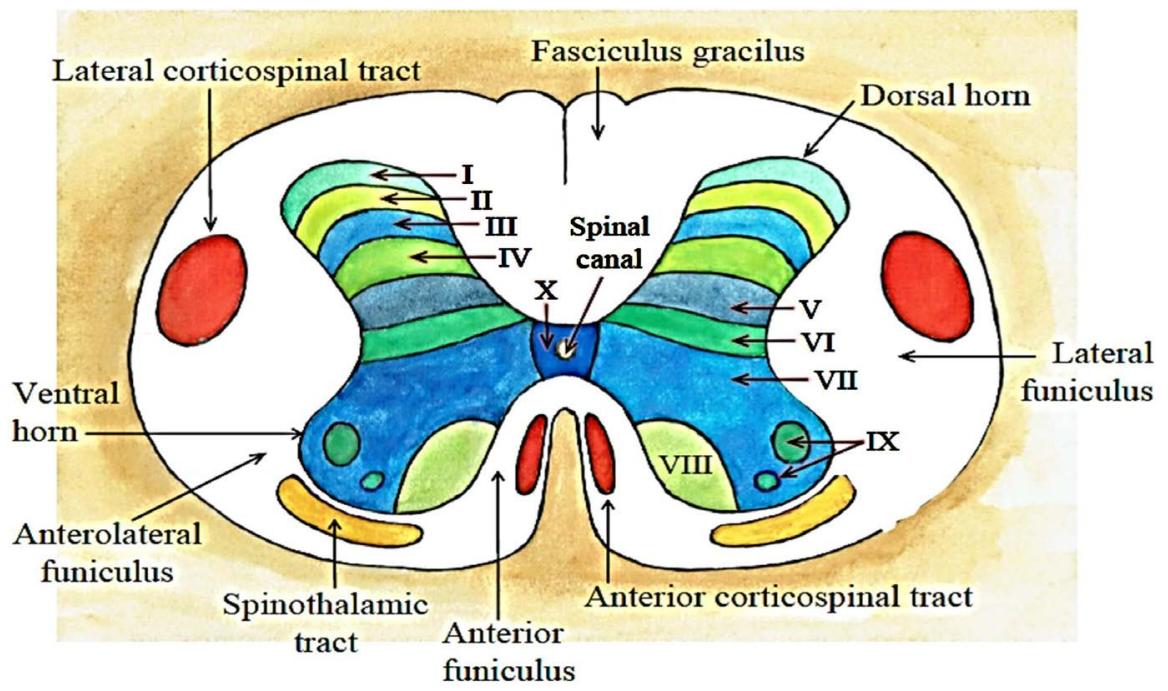

Figure 10. Lamina I, II, III, IV, V, VI, VII, VIII, IX, and X of the grey matter are labelled. The yellow small circle at the center is the spinal canal through which the cerebro-spinal fluid flows. Stimulating acupoint LU11 and another acupoint at the arm of the Lung Meridian send signals through the ascending spinothalamic tract (yellow-orange) to reach the thalamus. In response to the sensory signals, the motor neurons carry signals down the spinal cord along the anterior corticospinal tract and the lateral corticospinal tract respectively. The descening neurons send signals, via inter-neurons, to different parts of the respiratory system.

about perpendicular to the paper plane, and the descending "anterior corticospinal tract" (reddish) as well as "lateral corticospinal tract" (red) through which motor neurons descend. We can now proceed to follow two special stimulations along the Lung meridian.

\subsection{Stimulating the Acupoint LU11 and LU4 (An Acupoint on the Arm) Sends Signals along the Spinothalamic Tract to the Thalamus, Then to the Neocortex}

Now apply acupuncture/acupressure at the LU11 (shaoshang, thumb site) by perpendicular insertion 0.5 to $1 \mathrm{cun}$, and use the standard twirling technique to stimulate. We follow the signal along a sensory neuron (yellow) along the posterior spinal nerve T1 in Figure 11 (or C7, C8 of the cardiac-pulmonary plexus); the spinal neuron enters the posterior horn of grey matter (in this diagram the left side of the human body) as the first sensory neuron. It synapses the second neuron at this horn and the second neuron passes near the neighborhood of lamina $\mathrm{X}$ to the white matter column along the spinothalamic tract, which is well established (see, e.g., Fig. III.4 of [35]; Fig. 8.16 of [68]). Neurons along this tract synapse the second neurons at the grey horns of the $\mathrm{C} 3, \mathrm{C} 4$, and $\mathrm{C} 5$ as described in Section (4.3) with Figure 6.

Among these three spinal nerves, the $\mathrm{C} 4$ spinal nerve is the main one forming the phrenic nerve, which is the only motor nerve to move the diaphragm, without which, breathing will surely stop. At $\mathrm{T} 1$ (or C7/C8), after synapsing, the second neuron (also yellow) goes through the brain stem all the way up to the 


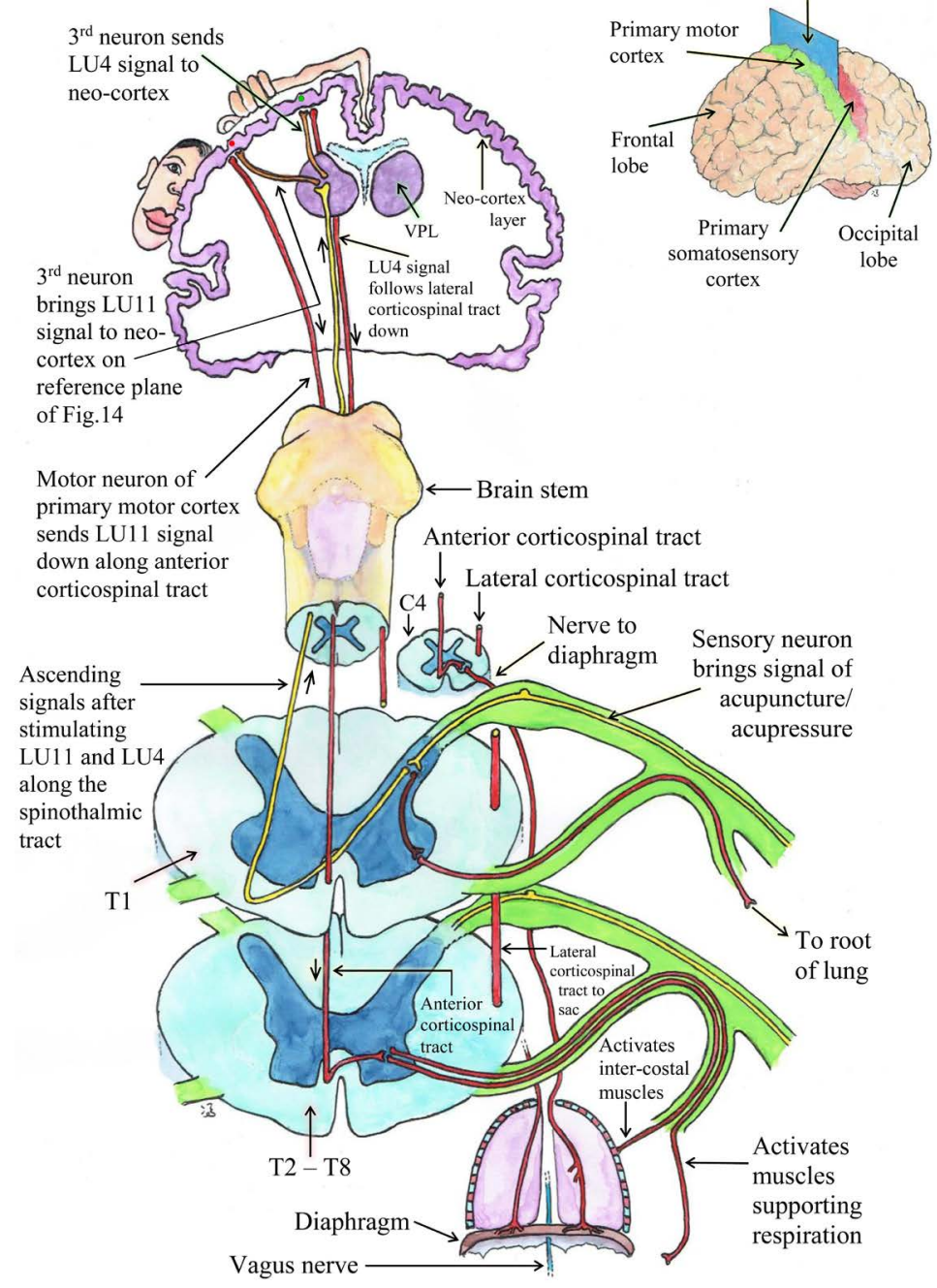

Figure 11. Ventral view of the neuronal system involved in responding to stimulation of the Lung Meridian. The yellow sensory neuron entering the dorsal grey horn comes from either one of the three nerves: 1) Median nerve; 2) Radial nerve; 3) Musculocutaneous nerve, following the procedure depicted in Figure 3. The afferent neurons in yellow form the spinothalamic tract, having its destination at the ventral posterior lateral (VPL) nucleus of the thalamus. At VPL, the third neuron (orange) synapses neurons at the neo-cortex. If stimulation at LU11 (shaoshang) is performed, the excited site (red dot) corresponds to the "thumb area". In response, the motor neuron follows the anterior corticospinal tract (red). This tract passes down through the anterior white matter sections. Then the neuron synapses the second neurons at the C3, C4, and C5 spinal grey horns. We show the second neuron at the $\mathrm{C} 4$ cord, joining the phrenic nerve [72]. The anterior corticospinal tract follows down through the cervical and thoracic spinal cords (see, e.g., Fig. III.29 of [35], Fig. 8.17 of [68]). This tract synapses other motor neurons at the ventral horns of the thoracic cords (T2-T8), leading to innervation of the intercostal muscles and the abdominal muscles supporting breathing [73]. If acupoint such as LU4 (xiobai) at the arm position is stimulated, the signal will be transmitted to reach the "arm site" (green dot) at the neo-cortex. The response follows the lateral corticospinal tract, which goes all the way down to the white matter of S1, S2, S3, and S4 (see Section (5.5)). The lateral corticospinal neuron also synapses motor neurons at the ventral horns in basically all the thoracic cords. These motor neurons innervate many muscles stripes, including those accessory muscles supporting breathing action in the abdominal region. The symbol "sac" represents sacrum. The motor neuron (red) exiting from the ventral horn of T1 in the middle of the Figure innervates the root of lung, as described in Section (4.6). Motor neuron from C6 (not shown) activates the scalene muscles which are needed for inspiration. The two-dimension view of the cortex corresponds to the sight looking at the primary somatosensory cortex (red) in the little figure on the upper right corner. The reference plane (blue) turns 90 degrees to become the two-dimension view of the cortex. If a neuron (pyramidal essentially) in the red region is to communicate with the associated neuron in the green region (primary motor cortex), it does not have to extend its collateral axons (Figure 1(d)) very far. This and all the figures of this paper were hand-painted by author PCWF. 
ventral posterior lateral (VPL) motor nucleus of the thalamus to synapse the third neuron (orange), whose destination is the neocortex layer ( $\sim 2 \mathrm{~mm}$ thick) of the primary somatosensory cortex corresponding to the thumb site (red dot) in the upper part of Figure 11 [69] [70]. In fact, the pioneer work of the limb-cortex connection in the cat model was published over half a century ago [71]. Suppose we stimulate another acupoint such as LU4 (xiabai) of the Lung Meridian on the arm, also by perpendicular insertion 0.5 to 1 cun, applying also the standard twirling technique. The ending spinothalamic path is different, but very close to the previous one, and the third sensory neuron will have its destination at another site, correspond to the "arm site" (green dot) in Figure 11.

It is important to note that the upper part of Figure 11 represents the cross-section of the brain specified by the blue plane in the little figure on the upper right corner. This plane lies between the primary somatosensory cortex (red) and the primary motor cortex (green).

\subsection{Evidence of the Cross-Talks between the Pyramidal Neurons in the Primary Somatosensory Cortex and the Primary Motor Cortex at the Fifth and Sixth Layers, in Response to Stimulation at Acupoints of the Lung Meridian}

The primary somatosensory (S1) neurons and primary motor (M1) neurons are long hypothesized to be reciprocally connected, and intuitively such hypothesis is sound, as animals and humans demonstrate that coordinated motor output is responsible to sensory input, for survival. That these connections are two-ways because the motor neurons can feed back their plan of motor action to the sensory neurons. Such intuition is supported by the experimental finding that discontinuation of S1 input to M1 would lead to disruption of many motor actions such as hand grip, chewing in primates and actions of other animals (see, e.g., [74] [75]). Note that the primary somatosensory cortex and the primary motor cortex are "neighbours' in the brain cortices (see upper right corner of Figure 11). A sensory neuron communicates with the related motor neuron in near distance ( $\sim 2 \mathrm{~mm}$ in the rat model). For humans, the communication distance is anticipated to be of the same scale of length. We will explain below how they "talk".

Refer to Figure 1(d), a pyramidal neuron has collateral axons (which are much longer than that shown in Figure 1(d) with respect to the length of the whole neuron) extending horizontally, implying that nature has prepared the ways for connections between "neighbouring pyramidal neurons (sideways)".

Recent work has shown that the probability of connection among pyramids is rather specific, meaning interactions are inhomogeneous [76]. Numerous experiments using animal models have been performed to illustrate such close connection, and it is also the basis for acupuncture/acupressure/moxibustion to function as therapeutic treatment to many disorders in muscles and internal organs. Here we review briefly several recent experimental results of such S1-M1 connection. Many sensory neurons from the peripheral synapse the third neurons in the thalamus, which have their destinations at one or more of the six 
cortical layers 1 to 6 as marked on the side of Figure 12 (divided along the length of $2 \mathrm{~mm}$ ) within the neo-cortex. The primary motor cortex also receives input signals from premotor, executive, and sensory centers [77] [78]. A special "sensorimotor slice preparation technique", which can preserve much of the S1-M1 connectivity has been developed, allowing in vitro studies on the anatomical structures of participants of such cross-talks. Photographs of the stained sensory and motor neurons at the neo-cortex have been reported in [79]. We will show in Figure 12 a hand-painted picture of the photography, i.e. Figure 2(A) of [79] (at the neo-cortex; the mouse whisker model has been a popular one to study sensory-motor communication, see, e.g. [80]). Such a photo shows clearly the communication between the sensory and motor neurons obtained by this special staining technique. The neurons appear red, and the horizontal thin lines are either the collateral axons or basal dendrites of the sensory and motor neurons within the neo-cortex layer. This direct evidence substantiates the electro-encephalography studies on the scalp, together with myo-graphic studies in the past several decades concerning sensory and motor neuron communication [81] [82] [83] [84] for humans in the awake states. We are confident to say that stimulation at the acupoints of the Lung Meridian can send signals to the primary motor region, which then send descending signals in response, as a natural physiological process.

\subsection{In Response to the Stimulation Action of the Acupoint LU11 of the Lung Meridian Described in Section (5.2), the Motor Neuron Follows the Anterior Cortico-Spinal Descending Pathway to Monitor Breathing}

We refer back to Figure 11. The descending motor neuron (reddish orange)

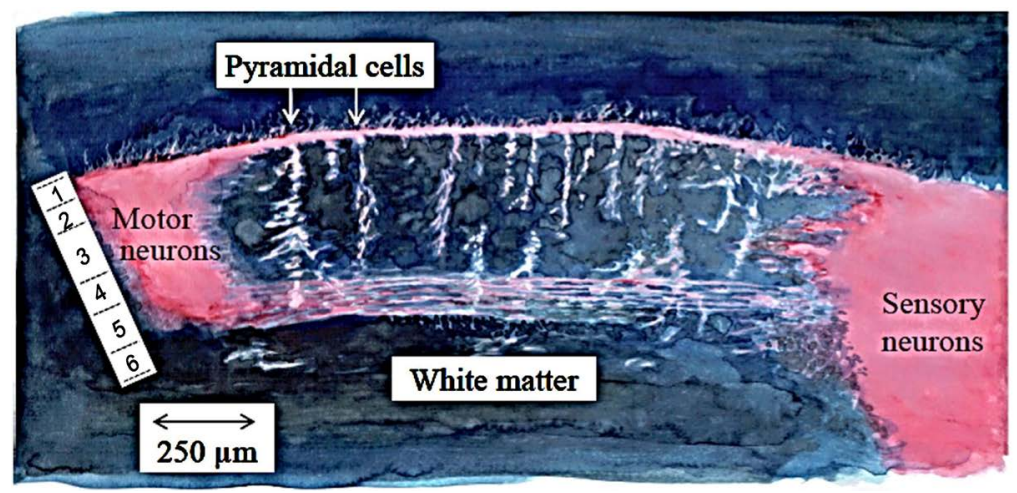

Figure 12. Schematic representation of the two-way communications between pyramidal neurons (of mice models) at the primary somatosensory neo-cortex (marked Sensory neurons) and that at the primary motor neo-cortex (marked Motor neurons). This is a hand-painted copy by author PCWF adapted from the photograph of ref [79]. Collateral axons of both sensory and motor neurons extended horizontally to communicate, as indicated by the thin horizontal red lines. Some isolated pyramidal cells are revealed between the two big groups of neurons. The pyramidal neurons of these two groups have their cell bodies mainly located in the $5^{\text {th }}$ and $6^{\text {th }}$ layers (marked 1 to 6 on the vertical side) of the neo-cortex, below which is the white matter. The scale is only a rough estimate. 
from the site corresponding to the thumb site (red dot) at the cortex goes down through the Pons, Medulla Oblongata and through the spinal cords, following the anterior corticospinal tract which synapses other neurons at the anterior/ventral grey horn of C3, C4, and C.5. The anterior corticospinal tract follows down through the cervical and thoracic spinal cords (see, e.g., Fig. III.29 of [35], Fig. 8.17 of [68]). In Figure 11, in the middle of the diagram, one sees, in the "downward direction", the cross-section of the C4 spinal cord. The second motor neuron from the left ventral grey horn of $\mathrm{C} 4$ follows the ventral spinal nerve to join the phrenic nerve.

Decades ago, there was direct evidence that motor neuron from the cortex can activate the diaphragm [85]. After "side-branching" to join the phrenic nerve, the anterior corticospinal tract is on route to C6, C7, C8 and then T1. From T1 to T11, the anterior corticospinal nerve synapses other motor neuron(s) at each of the T1-T11 cross-section, at the left ventral grey horn. We note, however, the motor neuron (red) exiting from the ventral horn of $\mathrm{T} 1$ in the middle of the Figure innervates the lung, as described in Section (4.6), with Figure 9.

Here we show a typical cross-section of the thoracic spinal cords T2-T8, in Figure 11. The activated neuron (reddish), which, via the ventral ramus, has its destination at the intercostal muscles (see Fig. 166 of [46]). The activated neurons at the left ventral grey horns of T2-T12 have their destinations at other accessory muscles of the body trunk, assisting the motion of the diaphragm [73].

\subsection{In Response to the Stimulation Action of the Acupoint LU4 of the Lung Meridian Described in Section (5.2), the Motor Neuron Follows the Lateral Corticospinal Tract Descending Pathway to Monitor Breathing, Plus Part of the Digestive System-We Provide Explanation of TCM Statement on "The Lung and the Large Intestine Are Inter-Connected"}

If one applies acupuncture or moxibustion at an acupoint (such as LU4) at the arm, the third motor neuron, after being synapsed at the ventral posterior lateral nucleus of the thalamus, would have its destination near the center of the parietal section (green dot in Figure 11), being different from the "thumb site" (red dot) of the motor cortex. The responding motor neuron descends from the cortex follows the lateral corticospinal tract (Fig. III.29 of [35]). This tract goes down the pons, crosses over to the left side at the decussation region of the Medulla Oblongata. We see a nerve emerging downwards in the left lateral white matter region in Figure 11. The very interesting aspect is that the lateral corticospinal tract has its destination at the spinal cords S1, S2, S3, and S4, discovered a few decades ago [86] [87].

In the guinea pig, it was established that from the inferior mesenteric ganglion (IMG) "sends off" sympathetic nerves innervating different bowel regions. Using the guinea pig model and retrograde labelling, the density of sympathetic nerves innervating the large intestine system was investigated [88]. By counting the number of neurons, it was concluded that IMG in the guinea pig receives a sig- 
nificant innervation of visceral sympathetic neurons from the middle, distal colon and maximizing at the rectum. In another study, analyzing the characteristics of the response of afferent nerve fibers innervating IMG associated with colonic distension in the guinea pig model also led to a very similar conclusion [89].

Aiming at the same purpose of analyzing nerves innervating the large intestine system of the rat model, a series of intramuscular injections were carried out with retrograde tracer cholera-toxin HRP (CT-HRP) [90] into the cecum, ascending, transverse, descending of the colon (except the rectum), to analyze the connection between the dorsal motor nucleus of the vagus nerve (DMN) and different portions of the large intestine [91]. The mean number of retrogradely labeled neurons in the DMN after injection to cecum, ascending colon, transverse colon, descending colon were respectively about 220, 90, 30, 20 (see Figure 3 of [91]). In this experiment with the rat model, there is clear evidence that the celiac and accessory celiac branches of the vagus nerves (as nerves of motor neurons) from the (lateral sides of) DMN innervate about the whole large intestine, but with decreasing influence towards the rectum (which is not innervated). Labelled neurons in the lumbo-sacral region of the spinal cord were reported to be revealed following injection to the descending colon and rectum. Result of this study, together with investigations such as that reported in [92], suggest strongly the innervation pattern presented in Figure 13, which summarizes the above findings. The yellow circles on the left represent the sympathetic ganglions on both anterior sides of the spine. The sympathetic trunk joins all these ganglions down to the pair corresponding to the Sacral 4 spinal segment. The celiac, the superior mesenteric, the inferior mesenteric ganglions (green circles) are "junctions" where the spinal nerves synapse the post-ganglionic neurons. The celiac branch of the parasympathetic nerve (blue) passes through the celiac ganglion and the superior mesenteric ganglion and innervates the small intestine (pink), as well as the ascending colon, cecum and transverse colon. The post-ganglionic neurons (orange) of the sympathetic nerves from the superior ganglion also innervate the ascending colon, cecum, and transverse colon. The post-ganglionic neuron from the inferior mesenteric ganglion, on the other hand, innervates the descending colon. Another sympathetic post-ganglionic neuron innervates the rectum. Parasympathetic nerves exiting from the S2, S3, S4 pass through the pelvic plexus and have a branch helping to tone the rectum and the descending colon.

As we follow the signals triggered by activation of the Lung Meridian, we observe that the celiac branch of parasympathetic nerve, passing through the diaphragm to tone three portions of the large intestine system, excluding the rectum and the descending colon. Based on the experimental studies reviewed in this section, activation of the inter-neuron at the grey matter at the center of the C3-T1 spinal cords would send signals to lamina X of the Medulla of the brain stem. The efferent nervous signals pass down to the sacral region where the parasympathetic nerves exit from the pelvic plexus to influence the action of the 


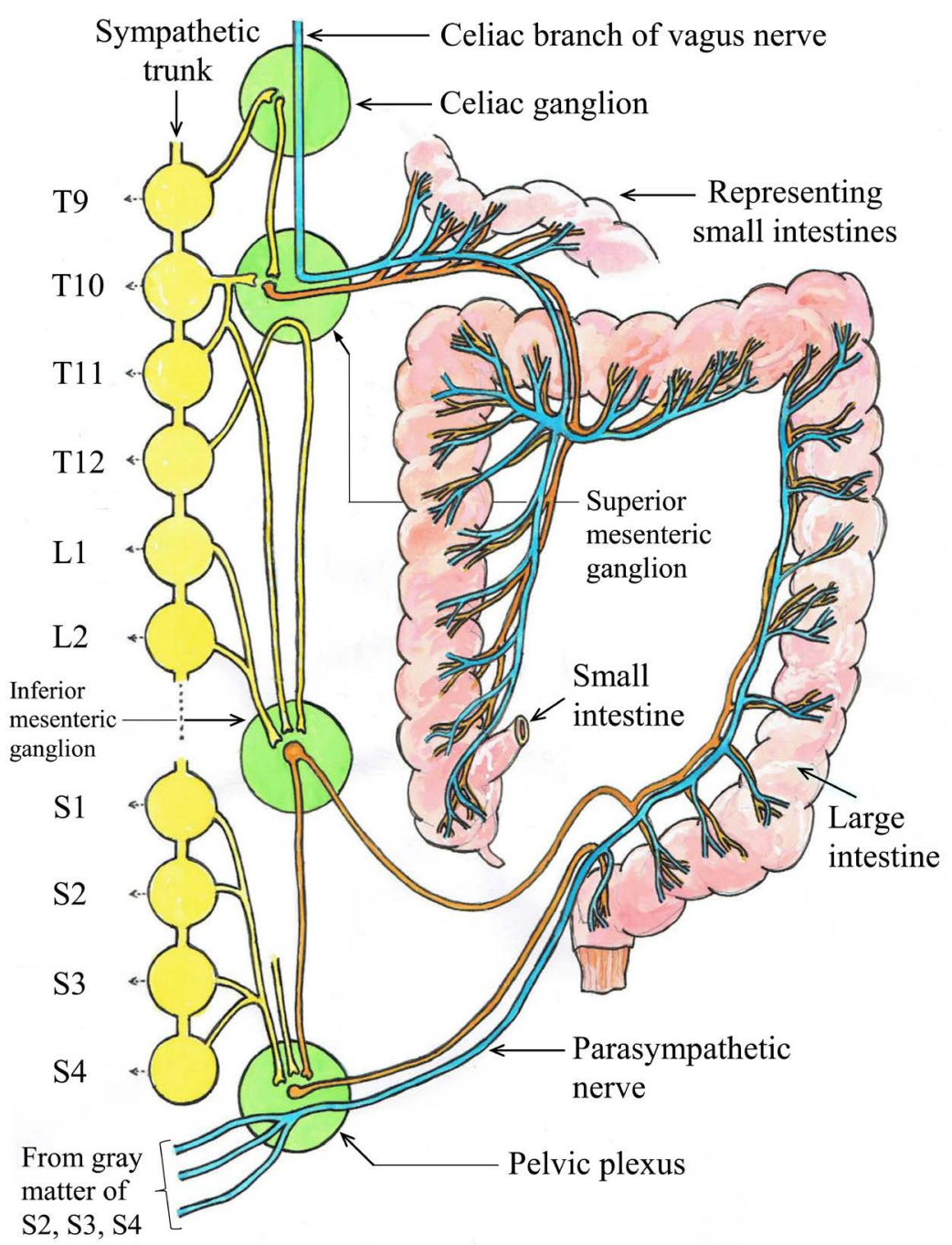

Figure 13. Innervation of the large intestine system. The yellow circles represent the sympathetic ganglions along the sympathetic trunk. L1, L2 are the two of the five lumber ganglions, whereas S1-S4 indicate schematically the positions of the sacral spinal segments from which the parasympathetic neurons (blue) exit. The green circles represent the ganglions where pre-ganglionic neurons synapse the post-ganglionic neurons. The vagus nerve follows lamina $\mathrm{X}$ all the way down the spinal cord (see Figure 10). This diagram is hand-painted adaptation of the figure on page 243 of [87] by author PCWF.

ascending colon, the rectum and the genital organ. It is remarkable that a few thousand years ago, in Traditional Chinese Medicine literature, the medical statement such as "the lungs are the external organ which connects the internal organ-the large intestine" already existed (see chapter 47.17 and 47.19 of [93]). There is also recent development of such connection [94] [95].

\subsection{The Respiratory Centers at the Pons, Medullar Oblongata and the Bulbospinal Tract System Automatically Generate Rhythmic Breathing Action}

Using in situ perfused (brain stem)-spinal cord specimens of the juvenile rat, the 
electric discharge pattern in real time were measured at the central vagus nerve (cranial X), hypoglossal nerve (cranial nerve IX), the pre-Bötzinger compartments of neurons [96] [97]. Such a study, plus other analyses demonstrated that there are basically three breathing centers in the Pons-medulla oblongata region: 1) The ventral respiratory group (VRG); 2) the dorsal respiratory group (DRG); 3) the Pontine center, as schematically shown in Figure 14. We accept the concept of [96] and consider the VRG to be composed of five groups of neurons [97]: a) the para-facial respiratory group (pFRG [98], also called the retrotrapezoid nucleus); b) Bötzinger complex (expiratory neurons); c) pre-Bötzinger complex (inspiratory neurons); d) rostral ventral respiratory group (rVRG, inpiratory neurons); e) caudal ventral respiratory group (cVRG, expiratory neurons).Whereas rVRG neurons project along the bulbospinal tract, the cVRG projects to thoracic and abdominal spinal expiratory motoneurons. The compartment of neurons called nucleus ambiguous (NA), is a continuation (upward) of a group of cells in the ventral horn of the spinal cord C1. The motor fibers of the cranial nerve IX, cranial nerve $\mathrm{X}$ exit the brain stem along and close to NA. Note that the dorsal respiratory group also lies close to the nucleus tractus solitarius (solitary tract nucleus), which is the domain where visceral afferents from cranial nerves IX and X terminate. These cranial nerves IX and X supply the striated muscle of the larynx and pharynx (discussed briefly in Section (4.5) with Figure 8). The pFRG compartment, which is just next to the VRG up the stem (may be considered as a continuation of the VRG) is lying close to the site where the facial nerve VII exits the brain stem to innervate the mucosal nasal gland (see Section (4.4) with Figure 7).

The general structure of the respiratory centers is summarized in Figure 14. The cranial nerves (parasympathetic) are painted in blue and the spinal nerves $\mathrm{C} 1$ and $\mathrm{C} 2$ are painted in yellow. NA is along the VRG for about the whole length. We have identified the VRG and DRG above. The pontine group (which is consisted of the Kölliker-Fuse nucleus, the lateral and medial parabrachial nuclei) can be simplified to be consisted of the parabrachial complex, including the lateral parabrachial nucleus and the medial parabrachial nucleus. Conventionally, many would consider the VRG controls breathing rhythm; however, according to the analysis of [97], it is more likely that the whole circuit constituting the VRG, DRG and Pons respiratory center regulates the breathing rhythm as a network, including inputs from the peripherals at the DRG.

The column of neurons in the pons-medullar-oblongata region joins the spinal cords as the bulbospinal tract system (greyish region bounded by the big dotted arrow).The bulbospinal tract system in literature is considered to be possibly composed of three pathways (A) The reticulospinal tract that passes through the anterior funiculus (Figure 10) close to the ascending spinothalamic tract; (B) the vestibulospinal tract which descend down through the anterolateral funiculus; (C) the rubrospinal tract which descends down the lateral funiculus (Figure III.37 of [35]). While tract (A) is established to be relating to respiratory 


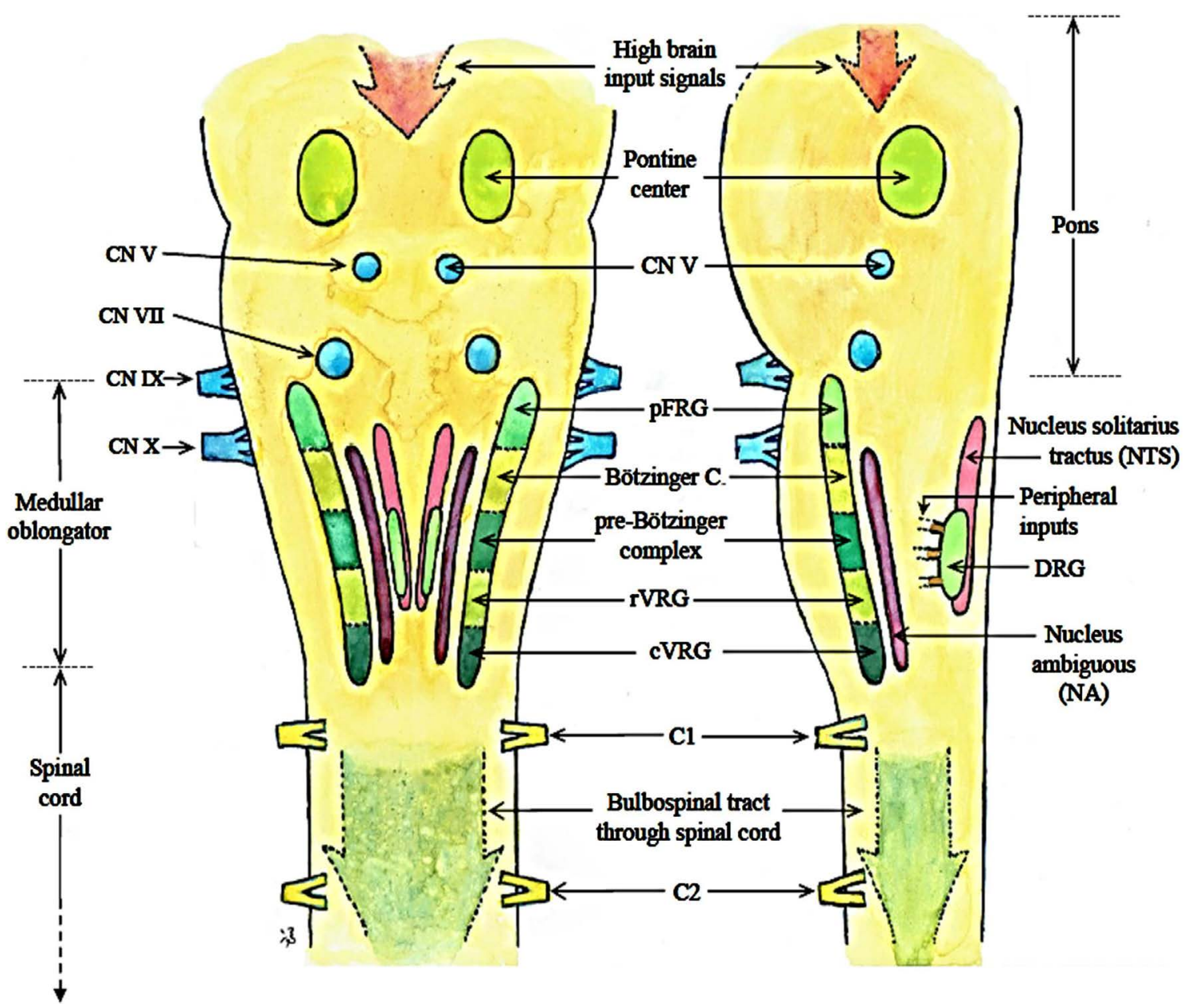

Figure 14. Schematic ventral and side views of the respiratory centers at the pons and medullar oblongata, and part of the spinal cords, showing the cranial nerves (blue) and sympathetic nerves (yellow). CN IX becomes glossolaryngeal nerve which innervates the larynx and pharynx (see Figure 8, Section (4.5)). The ventral respiratory center is composed of 5 compartments as described in the text. The column of neurons in the brain stem joins the spinal cords as the bulbospinal tract system (greyish region bounded by the big dotted arrow) [104]. There are input signals from the higher brain because the "thalamus-limbic-respiratory centers" circuit is at work [105]. DRG represents the dorsal respiratory group where input signals, (such as chemical signals) from the peripheral, enter. The nucleus tracts solitarius is an organization center of cardiac function [106], substantiating the close heart-lung relation. The nucleus ambiguous is the station where nerve CN X and nerve CN IX exit (see also Section (4.5) and Figure 8).

function, whether tracts (B) and/or (C) are involved in the automatic control of breathing was under research for some decades. However, it was found that after high cervical percutaneous cordotomy to treat cancer, all the patients died of respiratory dysfunction syndrome had lesions in the anterolateral funiculus in the $\mathrm{C} 2$ segment [72]. There was also direct evidence based electrical stimulation and subsequence analysis of the cat model that tract (B) participated in both respiratory and cardiac functions [99]. As tract (B) passes through anterolateral funiculus, one possibility of the result of [72] is that tracts (A) and (B) builds up the bulbospinal tract system.

The red arrow on top of the diagram represents there are input signals from the 
higher brain because the "thalamus-limbic-respiratory centers" circuit is at work [100]. Based on the detailed review in this Section, we can then state that the respiratory centers at the pons and medulla oblongata, working as a combined system, generate intrinsically a rhythmic respiratory rate and the neurons along the bulbospinal tract system, via tracts (A) and (B), are responsible to synapse the motor neurons (via interneurons in the grey regions of the spinal cords) of the sympathetic motor neurons to operate the muscles of the breathing system [100].

We must add a few words on the special neurons building up the bulbospinal tract system. We would emphasize that the neurons that innervate the spinal sympathetic preganglionic neurons (SPGNs), which build up the bulbospinal tract system, are called pre-sympathetic neurons. They are found in the upper cervical spinal cords, medulla oblongata, pons and the hypothalamus [101]. Research in the past decade gives complying evidence that they are very special neurons orchestrating many reflexes which are important to homeostasis such as keeping the respiratory rate, maintaining blood pressure and body temperature within the survival range [102]. These are the neurons that form tract (A) and (B) of the bulbospinal tract system mentioned above.

We wish to note one interesting in vitro experiment [103] relevant to our study. The brain stem and spinal cords were isolated from new born (0 - 4 days old) rat models. These preparations retained the thorax with innervated intercostal musculature. Also, the cervical and thoracic dorsal roots, the ventral spinal roots innervating the thoracic musculature were all kept intact for studying the respiratory function in a bathing solution. Suction electrodes were applied to the cut ends of spinal and cranial nerve ventral roots containing respiratory motoneuron axons; in this way the electrical activity is represented by the motor neuron discharge rates. Figure 15 (a hand-drawn painting by author PCWF, as an adaptation after Figure 1 of [103]) demonstrates the patterns of electric discharges of the motor neurons of the cranial nerves $\mathrm{CN}$ IX, CN X, as well as the electric discharge patterns of the sympathetic neurons at C1, C4, T2, T8 [103]. We observe that all these discharge patterns were found to be synchronized automatically once the vagus nerve triggered the respiratory system. In vivo electromyography measurements of the phrenic nerve, the diaphragm, and intercostal muscles ( $8^{\text {th }}$ space) were also carried out and the results were reported to substantiate the in vitro outcome.

Based on the above analysis, we know that a very sophisticated system is at work, including the cranial neurons, the pre-sympathetic neurons, and the sympathetic motor neurons, just to achieve smooth and non-stop breathing.

\subsection{Evidence of the Thalamus-Limbic-Respiratory Centers Circuitry Based on Functional Magnetic Resonance Imaging and Positron Emission Tomography Studies with Reference to Whether Stimulating the Lung Meridian Would Affect Emotion-A General Answer Was Documented over 1600 Years Ago}

There are pacemaker neurons (similar to those in the heart muscles) in the 

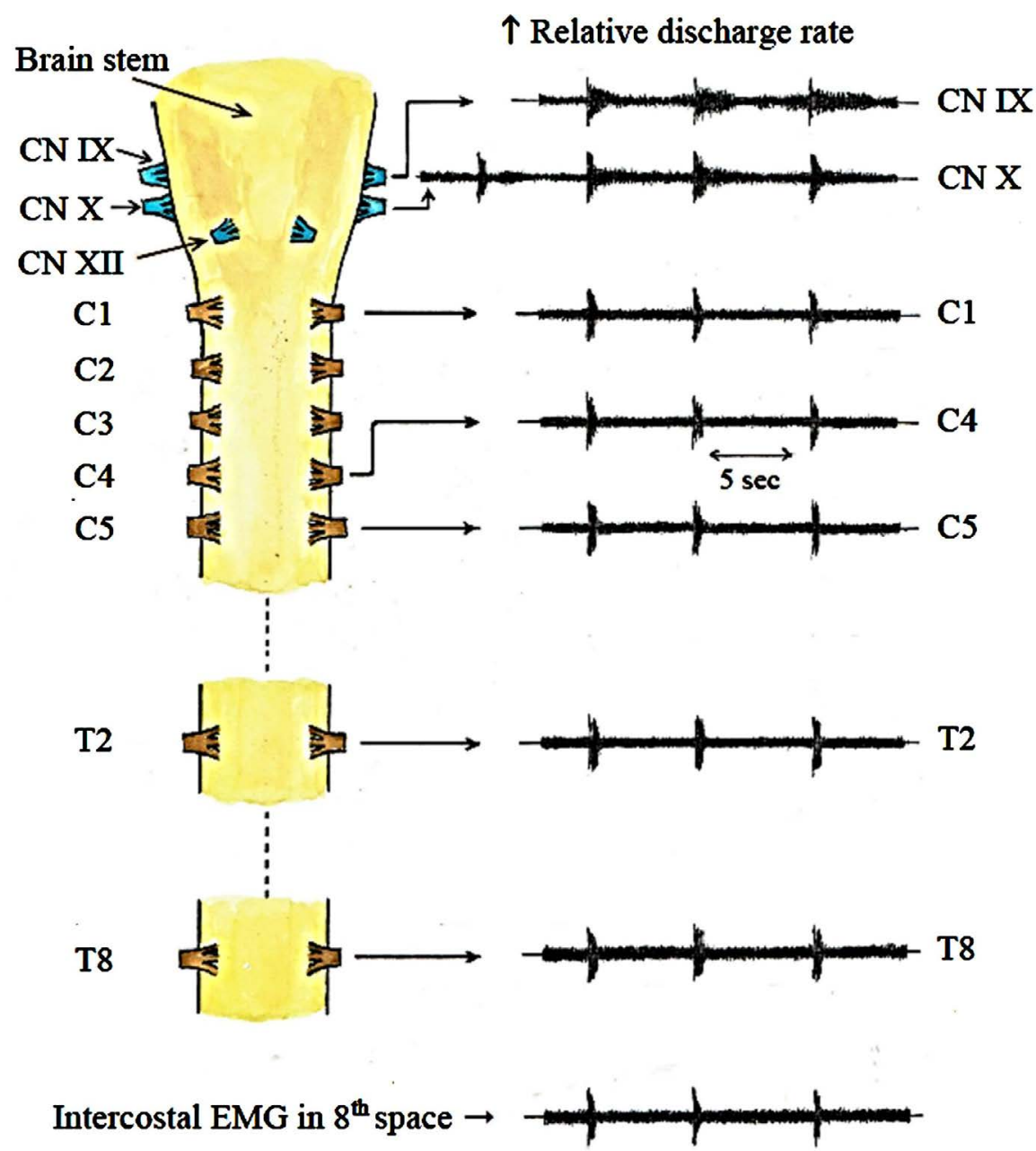

Figure 15. A rough hand-painting by author PCWF, as an adaptation of Figure 1 of [103] to show the electric discharge patterns of the motor neurons of the cranial nerves CN IX, CN X, sympathetic neurons at C1, C4, C5, T2, T8 of the rat model. All the discharged patterns in real time were found to be synchronized automatically once the vagus nerve triggers the respiratory system. In vivo electromyography pattern of the intercostal muscles ( $8^{\text {th }}$ space) is also shown.

brainstem providing automatic control of breathing for metabolic homeostasis, giving rise to an intrinsic brainstem respiratory rhythm to be controlled by a special network as depicted in the last Section. During the wake state, cognitive, emotion, physical demands modulate this rhythm for obvious reasons. Using functional magnetic resonance imaging (fMRI), the hemodynamics of the brain was recorded and other physical parameters (heart rate, respiratory rate, tidal volume, end-tidal volume of $\mathrm{CO}_{2}$ ) were measured simultaneously when 14 subjects were at rest [107]. This gave the control data set (i). This set of data suggests that there is synchronized neural activity across a distributed network of limbic/paralimbic and brainstem elements during resting condition. The subjects participated in a cognitive task (sustained random number generation) and the same recordings were carried out; data set (ii) were obtained when participants performed these tasks. Analysis of the two data sets (i) and (ii) indicate 
that there is coordinated neural activity associated with spontaneous breathing within the medulla, pons, midbrain, amygdala, anterior cingulate and anterior insular cortices. The active physical regions performing the neuro-modulation were found to be around the dorsal anterior cingulate and pontine raphe magnus nucleus. The authors propose that such active regions form a "limbic/ paralimbic-bulbar circuitry" which plays a significant role in cognitive and emotional modulation of spontaneous breathing [107] [108]. There is evidence of distributed brainstem and cortico-limbic responses to even short breath-holds [109]. In a recent study, the respiratory rate (RR) of 16 subjects were measured-these subjects were classified as the high trait (HT) anxiety and high state (HS) anxiety groups. Inspiratory $\left(\mathrm{T}_{\mathrm{I}}\right)$ and expiratory time $\left(\mathrm{T}_{\mathrm{E}}\right)$ were significantly shorter in both the HT and HS anxiety groups. The authors suggest that respiratory rhythm (reflected by the RR value) is affected by the degree of anxiety generated in the higher brain centers during quiet breathing in awake humans [110].

If the rhythmic breathing center is too sensitive to cognitive or emotional input, due to malfunction of some neurons in that center, it is not difficult to see that pathological states may result. It is thus interesting to find out whether in TCM, activation of acupoints of the Lung Meridian can be used to treat disorders related to emotion. In fact, acupuncture applied on the Lung Meridian was documented in Chapter 22.10 of [93], over 1600 years ago in TCM, to treat mood disorder. We have already explained clearly that stimulating the Lung Meridian would send signals to the VPL of thalamus via the spinothalamic tract. In view of the discovery of the Thalamus-Limbic-Respiratory Centers circuitry just mentioned in modern neurophysiology, it is not difficult to see that in general such signal would interfere with the stated circuitry relating mood. The particulars of the mechanism involve is yet unclear, and will be a fruitful research project, bridging the East and the West medicines.

\section{Discussion and Concluding Remarks-Bridging the East and West}

According to [1], chronic obstructive pulmonary diseases (COPD) caused 3.2 million deaths worldwide in 2015. It has been estimated in [111] that $8.2 \%$ of Chinese people over the age of 40 acquire COPD, and this disease is estimated to be the third highest cause of deaths worldwide by 2020. Various acupuncture, cupping, and herbal treatments have been tried in China, including applying acupuncture at one Lung acupoint, (Lei Que, see e.g. [112] [113]). As pointed out in [114], academic/clinical reports on the application of acupuncture have been focused on the efficacies of various choices of acupoints/treatments, without presenting analysis of the mechanisms behind those treatments. Without understanding the basic mechanisms behind any medical treatment is the bottle-neck to advancement of possibly effective therapy of any kind. Based on this argument, we start off a series of studies on the neurophysiological consequence of acupuncture/acupressure applied to one Meridian-the Lung Meridian (which is 
anticipated to be related to respiratory function according to TCM), because neuronal response is the most direct consequence of acupuncture/acupressure, which causes the sensation of dull-pain and pressure. Note also that nerve fibers accompanying blood vessels, lymphatic vessels, prevail in the interstitial fluid, and they innervate the proximity of all organs. All these types of fibers are enclosed by connective tissues built of collagen fibers and acupuncture/acupressure would therefore affect the properties of the blood and lymphatic vessels [2]. The references cited in the present paper cover a wide range in time epoch, because gross anatomy and some basic neurophysiology were developed half a century ago. This is a multi-disciplinary project, and we inevitably need to introduce some basics of neurophysiology, for convenience of the readers.

We have shown, using the "neuron-to-neuron tracking" approach, by taking evidence from either in vitro (animal models) studies or in vivo (animal models analysis plus some clinical case reports) investigations, that stimulating the Lung Meridian would affect the functions of organs A1 - A7 (Section 1.2) involved directly in breathing. To ease off the complication in analysis, we have only considered stimulating acupoints LU11 and LU4 as two examples, without loss of much generality. The key neuro-tracts involved are the spinothalamic tract, anterior corticospinal tract, the lateral corticospinal tract. We have explained the seeming mystery in TCM how the functions of the lung and the large intestines are connected in detail-such statement on interconnection was already documented in TCM literature over 1600 years ago [93].

Note that the connection between sensory stimulation and the function of internal organs using experimental techniques in modern biomedical science was reported as somato-visceral organ reflexes which could involve nerves innervating spinal cords. Some of the reflexes involve nerves in the brain together with some nerves in the spinal cords [115]. These studies show that stimulating sensory nerves in some regions of the animal models would either enhance or inhibit the functions of internal organs (like intestine mobility). The verifications of these somato-visceral organ reflexes did not refer to the special properties of the Meridians. We have pointed out in the series of recent analyses that the density of sensors is higher at acupoints [41] and the Meridian is a more efficient communication tract (as compared to non-meridian regions) in many aspects as outlined in [2] [8] [9]. We therefore anticipate that somato-viseral organ reflexes would be more efficient along the Meridians.

We have reviewed briefly the neuro-circuitry of providing automatic generation of the breathing rhythmic function during the wake and sleep state in Section (5.6) based on modern neurophysiology. There is dysfunction of respiration if any part of the circuitry is defective in the pathological state. The respiratory system, like heart beating, would be fatal if it stops function. Since the natural automatic respiration works on pathways different from the tracts resulting from stimulating the Lung Meridian as analyzed in previous sections, we propose that non-invasive (acupressure/moxibustion) or minimal invasive (like acupuncture) would help to restore respiration in COPD, as matter of principle. However, we 
do not know whether the motor neuron from the cortex through the lateral corticospinal tract or the anterior corticospinal tract (responding to acupuncture of the Lung Meridian) has connection with the bulbospinal tract system at the brain stem. If the answer is positive, there is "double benefit" by simulating the Lung Meridian to strengthen breathing action. Intuitively, exercise mobilizes motor neurons in general, and breathing is experienced to be smooth during exercise within personal limits. Having understood the basic mechanisms in affecting the organs involved in the respiratory function behind the action of stimulating the Lung Meridian, the next step is to work on particulars of treatment techniques. Along this line, we wish to note in a recent paper [9] that the TCM Sinew Channel and Meridian are closely related anatomically. Fluent blood, interstitial fluid and lymph flows, relaxation of the relevant connective tissues and muscles are also involved, as briefly stated in [9] for good communication along the Meridian. A follow-up analysis involving muscles of the Sinew Channel is necessary for finding concretely the suitable techniques to treat disorders of the lungs, as a second step-such an issue is the content of the next paper to come. In fact, there are quite a number of parameters involved to specify the disease states such as sleep apnea, as a special case of COPD [116], and the specific TCM technique to be used is disease-dependent. The choice of the two acupoints LU11 and LU4, based on the analysis in the previous sections, is only a starting point, with the standard techniques of stimulation stated in Section 5.2. We wish to remark also that it is common in TCM to stimulate a number of acupoints of different Meridians to treat the disease of a single organ [117]. Relating to the function of lungs, the acupoint feishu (BL13) of the Urinary Bladder Meridian has been used to treat certain lung disorder [112]. From the view point of neuro-physiology, certainly a set of nerves, different from those discussed in this paper, are involved when BL13 is stimulated. We consider stimulating BL13 would affect the lung function via the posterior roots of the lungs due to obvious anatomical reason. We only analyze one Meridian at a time. After analyzing the neurophysiological consequences of all the twelve Meridians plus the related Sinew Channels, we will see the overall picture of the efficacy of acupuncture/acupressure. The modality of treatment has to be adjusted as we accumulate more knowledge of the neurophysiological responses, directly (from in vivo studies) and indirectly (from in vitro investigations).

On the other hand, we have not discussed the effects of moxibustion in this paper, because even mild hyperthermia caused the activation of Heat Shock Proteins which participate in many physiological pathways as analyzed in our recent paper (see Figure 2 of [118]); this issue of moxibustion deserves another series of papers.

Moreover, the recent discovery of the Thalamus-Limbic-Respiratory Centers circuitry points to the fact that respiration is closely related to change of mood. Thus stimulating the Lung meridian "branches" out as another interesting and useful issue for mood regulation, which is of interest to psychology and psychiatry. A pioneering work (at modern time) on applying acuputure at LU9 
(Taiyuan) has recently been found to be correlated to increased cerebral hemodynamic activities at the subcallosal gyrus, right inferior frontal gyrus plus other cerebral regions [119]. Such enhancement could well be triggered off by the sensory signal (traveling along the spinothalamic tract) reaching the VLP of thalamus, which is a center of activities, as mentioned in this paper.

\section{Conclusion}

Stimulating the Lung Meridian via sensory neurons would activate the organs A1 - A7 involved in respiration in ways different from the intrinsic pathways through the respiratory centers. We put forth the notion that, together with the toning of the Lung Sinew Channel using special manual maneuver before applying acupuncture (to be published in the next paper), modernized non-invasive TCM modalities might provide the chance of sustaining continuous respiration when the intrinsic system is defective in some cases of COPD. The details of maneuver techniques, and clinical trials are disease dependent and waits for a series of studies hopefully to be carried out by different groups in the near future as mentioned in the early part of this section. Monitoring the mood, functions of the large intestine and sweating via stimulation of the Lung Meridian are also interesting projects with clinical relevance.

\section{Acknowledgements}

Hearty thanks are due to Mr. Benjamin Fung, brother of PCWF, for his unfailing support and assistance during the preparation of this manuscript. We wish to thank Miss Yuefei Ruan for her assistance also during the preparation of the manuscript. All the Figures were hand-painted by author PCWF. There is no conflict of interests in this academic project.

\section{References}

[1] Chronic Respiratory Disease Collaborators (2017) Global, Regional, and National Deaths, Prevalence, Disability-Adjusted Life Years, and Years Lived with Disability for Chronic Obstructive Pulmonary Disease and Asthma, 1990-2015: A Systematic Analysis for the Global Burden of Disease Study. The Lancet, 5, 691-706.

[2] Fung, P.C.W. (2013) Plausible Biomedical Consequences of Acupuncture Applied at Sites Characteristic of Acupoints in the Connective-Tissue-Interstitial-Fluid System. In: Chen, L.L. and Cheng, T.O., Eds., Acupuncture in Modern Medicine, Chapter 5, IntechOpen, Rijeka, 95-131. https://doi.org/10.5772/53901

[3] Fung, P.C.W. and Kong, R.K.C. (2016) The Integrative Five-Fluid Circulation System in the Human Body. Open Journal of Molecular and Integrative Physiology, 6, 45-97. https://doi.org/10.4236/ojmip.2016.64005

[4] Dang, R.S., Chen, E.Y., Shen, X.Y., et al. (1997) Relation of Connective Tissue to the Acupoints of the Lung Meridian. Shanghai Zhenjiu (Shanghai Journal of Acupuncture and Moxibustion), No. 4, 28-29.

[5] Fei, L., Cheng, H.S., Cai, D.H., Yang, S.X., Xu, J.R., Chen, E.Y., Dang, R.S., Ding, G.H., Shen, X.Y. and Tang, Y. (1998) Experimental Investigation and Scientific Demonstration of the Materialistic Foundation of Meridians and Their Functional 
Specialties. Kexue Tongbao, 43, 658-672.

[6] Yuan, L., Yao, D.W., Tang, L., Huang, W.H., Jiao, P.F., Lu, Y.T., Dai, J.X., Zhang, H., He, Z.Q. and Zhong, S.Z. (2000) A Study on Morphological Basis of Chinese Acupuncture and Moxibustion from Digital Human Body. Jiepou Xuebao (Acta Anatomica Sinica), 35, 337-343.

[7] Langevin, H.M. and Yandow, J.A. (2002) Relationship of Acupuncture Points and Meridians to Connective Tissue Planes. The Anatomical Record, 269, 257-265. https://doi.org/10.1002/ar.10185

[8] Fung, P.C.W. (2009) Probing the Mystery of Chinese Medicine Meridian Channels with Special Emphasis on the Connective Tissue Interstitial Fluid System, Mechanotransduction, Cells Durotaxis and Mast Cell Degranulation. Chinese Medicine, 4, 10. https://doi.org/10.1186/1749-8546-4-10

[9] Fung, P.C.W. and Kong, R.K.C. (2018) Relationship among the Meridians, Sinew channels and Integrative Five Fluid Circulation System. Traditional Chinese Medicine, 7, 74-92. https://doi.org/10.12677/TCM.2018.71013

[10] Novotny, G.E.K. and Gnoth, C. (1991) Variability of Fibroblast Morphology in Vivo: A Silver Impregnation Study on Human Digital Dermis and Subcutis. Journal of Anatomy, 177, 195-207.

[11] Schwarzbauer, J.E. and Sechler, J.L. (1999) Fibronectin Fibrillogenesis: A Paradigm for Extracellular Matrix Assembly. Current Opinion in Cell Biology, 11, 622-627. https://doi.org/10.1016/S0955-0674(99)00017-4

[12] Turksen, K. (2004) Adult Stem Cells. Humana Press, Totowa, NJ. https://doi.org/10.1007/978-1-59259-732-1

[13] Tenney, R.M. and Discher, D.E. (2009) Stem Cell, Microenvironment Mechanics, and Growth Factor Activation. Current Opinion in cell Biology, 21, 630-635. https://doi.org/10.1016/j.ceb.2009.06.003

[14] Wing, S.T. and Kim, W.S. (2011) Plasma and Cellular Fibronectin: Distinct and Independent Functions during Tissue Repair. Fibrogenesis and Tissue Repair, 4, 21. https://doi.org/10.1186/1755-1536-4-21

[15] Zhu, Z. and Xu, R. (1990) Morphometric Observation on the Mast Cells under the Acupuncture Meridian Lines. Zhen Ci Yan Jiu, 15, 157-158.

[16] Metcalfe, D.D., Baram, D. and Mekori, Y.A. (1997) Mast Cells. Physiological Reviews, 77, 1033-1079. https://doi.org/10.1152/physrev.1997.77.4.1033

[17] Maurer, M., Theoharides, T., Granstein, R.D., Bischoff, S.C., Bienenstock, J., Henz, B., Kovanen, P., Piliponsky, A.M., Kambe, N., Vliagoftis, H., Levi-Schaffer, F., Metz, M., Miyachi, Y., Befus, D., Forsythe, P., Kitamura, Y. and Galli, S. (2003) What Is the Physiological Function of Mast Cells? Experimental Dermatology, 12, 886-910. https://doi.org/10.1111/j.0906-6705.2003.0109a.x

[18] Gilfillan, A.M., Austin, S.J. and Metcalfe, D.D. (2011) Mast Cell Biology: Introduction and Overview. Advances in Experimental Medicine and Biology, 716, 2-12. https://doi.org/10.1007/978-1-4419-9533-9_1

[19] Yao, W., Yang, H., Yin, N. and Ding, G. (2014) Mast Cell-Nerve Cell Interaction at Acupoint: Modeling Mechanotransduction Pathway Induced by Acupuncture. International Journal of Biological Sciences, 10, 511-519. https://doi.org/10.7150/ijbs.8631

[20] Gil, H.J., Bae, K.H., Kim, L.J., Kim, S.C. and Soh, K.S. (2015) Number Density of Mast Cells in the Primo Nodes of Rats. Journal of Acupuncture and Meridian Studies, 8, 288-293. https://doi.org/10.1016/j.jams.2015.03.007 
[21] Jang, H.S., Yoon, J., Gil, H.J., Jung, S.J., Kim, M.S., Lee, J.K., Kim, Y.J. and Soh, K.S. (2016) Observation of a Flowing Duct in the Abdominal Wall by Using Nanoparticles. PLoS ONE, 11, e0150423. https://doi.org/10.1371/journal.pone.0150423

[22] Deng, Y., Zeng, T., Zhou, Y. and Guan, X. (1996) The Influence of Electro-Acupuncture on the Mast Cells in the Acupoints in Stomach Meridian. Zhen Ci Yan Jiu, No. 3, 68-70.

[23] Zhang, Y.J., Wang, X.Y., Sh, L.P., Miao, W.F., Xu, T.P. and Zhang, C.X. (1995) Influence of Changing $\mathrm{Ca}^{2+}$ Concentration in Neiguan (PC6) on the Effect of Acupuncture Treating Experimental Arrhythmic Rabbits. Zhenci Yanjiu (Acupuncture Research), No. 2, 63-67.

[24] Hu, L.M., Zhang, Y.J. and Zhang, C.X. (1998) The Changes of $\mathrm{Ca}^{2+}$ in Points along the Pericardium Channel and the Influence of Acupuncturing Neiguan. Zhenci Yanjiu (Acupuncture Research), No. 4, 251-253.

[25] Guo, Y., Ma, D.M., Zhang, C.X., Hu, L.M., Yuan, Y.J. and Wang, G.J. (2003) On the Concentration of Calcium Element in Zusanli (ST36) in Rabbits. Shanghai Zhenci Zha Zhi (Shanghai Journal of Acupuncture and Moxibustion), No. 7, 26-27.

[26] Ernst, M. and Lee, M.H. (1985) Sympathetic Vasomotor Changes Induced by Manual and Electrical Acupuncture of the Hoku Point Visualized by Thermography. Pain, 21, 25-33. https://doi.org/10.1016/0304-3959(85)90073-9

[27] Ulett, G.A., Han, S.P. and Han, J.S. (1998) Electroacupuncture: Mechanisms and Clinical Application. Biological Psychiatry, 137, 129-138. https://doi.org/10.1016/S0006-3223(97)00394-6

[28] Kawakita, K. and Okada, K. (2006) Mechanisms of Action of Acupuncture for Chronic Pain Relief-Polymodal Receptors Are the Key Candidates. Acupuncture Medicine, 24, S58-S66. https://doi.org/10.1136/aim.24.Suppl.58

[29] Wong, A.M.K., Su, T.Y., Tang, F.T., Cheng, P.T. and Liaw, M.Y. (1999) Clinical Trial of Electrical Acupuncture on Hemiplegic Stoke Patients. American Journal of Physical Medicine and Rehabilitation, 78, 117-122.

[30] Lee, H.S., Yu, Y.C., Kim, S.T. and Kim, K.S. (1997) Effects of Moxibustion on Blood Pressure and Renal Function in Spontaneously Hypertensive Rats. The American Journal of Chinese Medicine, 25, 21. https://doi.org/10.1142/S0192415X97000056

[31] Deng, H. and Shen, X. (2013) The Mechanism of Moxibustion: Ancient Theory and Modern Research. Evidence-Based Complementary and Alternative Medicine, 2013, Article ID: 379291.

[32] Langevin, H.M., Churchill, D.L., Wu, J., Badger, G.J., Yandow, J.A., Fox, J.R. and Krag, M.H. (2002) Evidence of Connective Tissue Involvement in Acupuncture. FASEB Journal, 16, No. 8. https://doi.org/10.1096/fj.01-0925fje

[33] Langevin, H.M., Bouffard, N.A., Churchill, D.L. and Badger, G.J (2007) Connective Fibroblast Response to Acupuncture: Dose-Dependent Effect of Bidirectional Needle Rotation. Journal of Alternative and Complementary Medicine, 13, 355-360. https://doi.org/10.1089/acm.2007.6351

[34] Langevin, H.M., Bouffard, N.A., Badger, G.J., Churchill, D.L. and Howe, A.K. (2006) Subcutaneous Tissue Fibroblast Cytoskeletal Remodeling Induced by Acupuncture: Evidence for a Mechanotranduction-Based Mechanism. Journal of Cell Physiology, 207, 767-774. https://doi.org/10.1002/jcp.20623

[35] Felten, D.L. and Jozefowicz, R.F. (2003) Netter's Atlas of Human Neuroscience. Icon Learning Systems, New Jersey.

[36] Micheva, K.D., Wolman, D., Mensh, B.D., Pax, E., Buchanan, J., Smith, S.J. and 
Bock, D.D. (2016) Axonal Myelination of Interneurons in Cortex: Functional Significance and Plasticity. eLife, 5, e15784.

[37] Castañeda-Corral, G., Jimenez-Andrade, J.M., Bloom, A.P., Taylor, R.N., Mantyh, W.G., Kaczmarska, M.J., Ghilardi, J.R. and Mantyh, P.W. (2011) The Majority of Myelinated and Unmyelinated Sensory Nerve Fibers that Innervate Bone Express the Tropomyosin Receptor Kinase A. Neuroscience, 178, 196-207. https://doi.org/10.1016/j.neuroscience.2011.01.039

[38] Gardner, E.P., Martin, J.H. and Jessell, T.M. (2000) The Bodily Senses. In: Kandel, E.R., Schwartz, J.H. and Jessell, T.M., Eds., Principles of Neural Science, Oxford University Press, New York, 430-449.

[39] Boron, W.F. and Boulpaep, E.L. (2016) Medical Physiology. E-Book, Elsevier Health Sciences, p. 384.

[40] Xu, W., Sun, G., Chen, X., Chen, Q., Fang, Q., Sun, N., Zhang, Y., Zhang, J. and Ren, M. (2009) Observation of Cranial Nerves in the Cerebellopontine Angle Region by Retrosigmoid Approach. Lin Chuang Er Bi Yan Hou Tou Jing Wai Ke Za Zhi (Journal of Clinical Otorhinolaryngology, Head, and Neck Surgery), 23, 454-455.

[41] Liu, K., Li, A.H., Wang, W. and Xie, Y.K. (2009) Dense Innervation of Acupoints and Its Easier Reflex Excitatory Character in Rats. Zhenci Yanjiu (Acupuncture Research), 34, 36-42.

[42] Tomassy, G.S., Berger, D.R., Chen, H.H., Kasthuri, N., Hayworth, K.J., Vercelli, A., Seung, H.S., Lichtman, J.W. and Arlotta, P. (2014) Distinct Profiles of Myelin Distribution along Single Axons of Pyramidal Neurons in the Neocortex. Science, 344, 319-324. https://doi.org/10.1126/science.1249766

[43] Regehr, W.G., Carey, M.R. and Best, A.R. (2009) Activity-Dependent Regulation of Synapses by Retrograde Messengers. Neuron, 63, 154-170. https://doi.org/10.1016/j.neuron.2009.06.021

[44] Brodal, P. (2004) The Central Nervous System: Structure and Function. 3rd Edition, Oxford University Press, US, 369-396.

[45] Diego, M.A. and Field, T. (2009) Moderate Pressure Massage Elicits a Parasympathetic Nervous System Response. International Journal of Neuroscience, 119 , 630-638. https://doi.org/10.1080/00207450802329605

[46] Clemente, C.D. (2006) Anatomy: A Regional Atlas of the Human Body. 5th Edition, Lippincott Williams and Wilkins, Los Angeles, CA.

[47] Deadman, P., Al-khafaji, M. and Baker K. (2015) A Manual of Acupuncture, California 92083, USA. Journal of Chinese Medicine Publications, Chapter of Lung Channel, 73-90.

[48] Brown, R.H. and Mitzner, W. (1996) Effect of Lung Inflation and Airway Muscle Tone on Airway Diameter in Vivo. The American Physiological Society, 80, 1581-1588. https://doi.org/10.1152/jappl.1996.80.5.1581

[49] Neurosurgery for Medical Students by Dr. Shyam Pujara. https://myneurosurg.com/

[50] Chandler, M.J., Zhang, J. and Foreman, R. D. (1996) Vagal, Sympathetic and Somatic Sensory Inputs to Upper Cervical (C1-C3) Spinothalamic Tract Neurons in Monkeys. Journal of Neurophysiology, 76, 2555-2567. https://doi.org/10.1152/jn.1996.76.4.2555

[51] Young, J., Finn, L. and Kim, H. (1997) Nasal Obstruction as a Risk Factor for Sleep-Disordered Breathing. The Journal of Allergy and Clinical Immunology, 99, S757-S762. 
[52] Heusch, G., Baumgart, D., Camici, P., Chilian, W., Gregorini, L., Hess, O., Indolfi, C. and Rimoldi, O. (2000) $\alpha$-Adrenergic Coronary Vasoconstriction and Myocardial Ischemia in Humans. Circulation, 101, 689-694. https://doi.org/10.1161/01.CIR.101.6.689

[53] Lung, M.A. (1995) The Role of the Autonomic Nerves in the Control of Nasal Circulation. Neurosignals, 4, 179-185. https://doi.org/10.1159/000109439

[54] Fuller, D.R., Pimentol, J.T. and Peregoy, B.M. (2012) Applied Anatomy and Physiology for Speech-Language Pathology and Audiology. Wolters Kluwer-Lippincott Williams and Wilkins, Baltimore, MD.

[55] Matsuo, K. and Palmerb, J.B. (2009) Coordination of Mastication, Swallowing and Breathing. Japanese Dental Science Review, 45, 31-40. https://doi.org/10.1016/j.jdsr.2009.03.004

[56] Schweizer, V. and Dörfl, J. (1997) The Anatomy of the Inferior Laryngeal Nerve. Clinical Otolaryngology and Allied Sciences, 22, 362-369. https://doi.org/10.1046/j.1365-2273.1997.00028.x

[57] Laitman, J.T., Noden, D.M. and Van De Water, T.R. (2006) Formation of the Larynx: From Homeobox Genes to Critical Periods. In: Rubin, J.S., Sataloff, R.T. and Korovin, G.S., Ed., Diagnosis and Treatment Voice Disorders, Plural, San Diego, 3-20.

[58] Troyer, A.D., Peche, R., Yernault, J.C. and Estenne, M. (1994) Neck Muscle Activity in Patients with Severe Chronic Obstructive Pulmonary Disease. American Journal of Respiratory and Critical Care Medicine, 150, No. 1. https://doi.org/10.1164/ajrccm.150.1.8025770

[59] Buford, J.A., Yoder, S.M., Heiss, D.G. and Chidley, J.V. (2002) Actions of the Scalene Muscles for Rotation of the Cervical Spine in Macaque and Human. The Journal of Orthopaedic and Sports Physical Therapy, 32, 488-496. https://doi.org/10.2519/jospt.2002.32.10.488

[60] Colebatch, H.J.H., Dawes, G.S., Goodwint, J.W. and Nadeaut, R.A. (1965) The Nervous Control of the Circulation in the Foetal and Newly Expanded Lungs of the Lamb. The Journal of Physiology, 178, 544-562. https://doi.org/10.1113/jphysiol.1965.sp007641

[61] Nagaishi, C. and Nagasawa, N. (1972) Anatomy, Surgical and Topographical. Chapter 1, University Park Press, Paddyfield.

[62] Evans Downing, S. and Lee, J.C. (1980) Nervous Control of the Pulmonary Circulation. Annual Review of Physiology, 42, 199-210. https://doi.org/10.1146/annurev.ph.42.030180.001215

[63] Cadieux, A., Monast, N.P., Pomerleau, F., Fournier, A. and Lanoue, C. (1999) Bronchoprotector Properties of Calcitonin Gene-Related Peptide in Guinea Pig and Human Airways Effect of Pulmonary Inflammation. American Journal of Respiratory and Critical Care, 159, 235-243. https://doi.org/10.1164/ajrccm.159.1.9711031

[64] Springall, D.R., Cadieux, A., Oliveira, H., Su, H., Royston, D. and Polak, J.M. (1987) Retrograde Tracing Shows that CGRP-Immunoreactive Nerves of Rat Trachea and Lung Originate from Vagal and Dorsal Root Ganglia. Autonomic Neuroscience-Basic and Clinical, 20, 155-166.

[65] Kummer, W., Fischer, A., Kurkowski, R. and Heym, C. (1992) The Sensory and Sympathetic Innervation of Guinea-Pig Lung and Trachea as Studied by Retrograde Neuronal Tracing and Double-Labelling Immunohistochemistry. Neuroscience, 49, 715-737. https://doi.org/10.1016/0306-4522(92)90239-X

[66] Shiev, D., Butler, J. and Lewis, R. (2010) Hole's Human Anatomy and Physiology. 
12th Edition, McGraw Hill, New York.

[67] Gibbins, I. (2013) The Human Nervous System. Chapter 5-Peripheral Autonomic Pathways, ScienceDirect, Elsevier, 141-185.

[68] Crossman, A.R. and Neary, D. (2010) Neuroanatomy. Elsevier, Churchill, Livingstone.

[69] Gauriau, C. and Bernar, J.F. (2002) Physiological Society Symposium Nociceptors as Homeostatic Afferents: Central Processing Pain Pathways and Parabrachial Circuits in the Rat. Experimental Physiology, 87, 251-258.

https://doi.org/10.1113/eph8702357

[70] Hooks, B.M., Mao, T., Gutnisky, D.A., Yamawaki, N., Svoboda, K. and Shepherd, G.M.G. (2013) Organization of Cortical and Thalamic Input to Pyramidal Neurons in Mouse Motor Cortex. Journal of Neuroscience, 33, 748-760. https://doi.org/10.1523/JNEUROSCI.4338-12.2013

[71] Oscarsson, O. and Rosin, I. (1966) Short-Latency Projections to the Cat Cerebral Cortex from Skin and Muscle Afferents in the Contralateral Forelimb. The Journal of Physiology, 182, 164-184. https://doi.org/10.1113/jphysiol.1966.sp007816

[72] Lahuerta, J., Buxton, P., Lipton, S. and Bowsher, D. (1992) The Location and Function of Respiratory Fibres in the Second Cervical Spinal Cord Segment: Respiratory Dysfunction Syndrome after Cervical Cordotomy. Journal of Neurology, Neurosurgery, and Psychiatry, 55, 1142-1145. https://doi.org/10.1136/jnnp.55.12.1142

[73] Blumenfeld, H. (2012) Neuroanatomy through Clinical Cases. First Edition, ISBN: 13:978-0878930609.

[74] Brochier, T., Boudreau, M.J., Paré, M. and Smith, A.M. (1999) The Effects of Muscimol Inactivation of Small Regions of Motor and Somatosensory Cortex on Independent Finger Movements and Force Control in the Precision Grip. Experimental Brain Research, 128, 31-40. https://doi.org/10.1007/s002210050814

[75] Hiraba, H. (2000) Deficits of Masticatory Movements Caused by Lesions in the Orofacial Somatosensory Cortex of the Awake Cat. Somatosensory and Motor Research, 17, 361-372. https://doi.org/10.1080/0899022002000202239

[76] Kampa, B.M., Letzkus, J.J. and Stuart, G.J. (2006) Cortical Feed-Forward Networks for Binding Different Streams of Sensory Information. Nature Neuroscience, 9, 1472-1473. https://doi.org/10.1038/nn1798

[77] Cicirata, F., Angaut, P., Cioni, M., Serapide, M.F. and Papale, A. (1986) Functional Organization of Thalamic Projections to the Motor Cortex. An Anatomical and Electrophysiological Study in the Rat. Neuroscience, 19, 81-99. https://doi.org/10.1016/0306-4522(86)90007-2

[78] Reep, R.L., Goodwin, G.S. and Corwir, J.V. (1990) Topographic Organization in the Corticocortical Connections of Medial Agrannular Cortex in Rats. JCN, 294, 262-280.

[79] Rocco, M.M. and Brumberg, J.C. (2007) The Sensorimotor Slice. Journal of Neuroscience Methods, 162, 139-147. https://doi.org/10.1016/j.jneumeth.2007.01.002

[80] Sreenivasan, V., Esmaeili, V., Kiritani, T., Galan, K., Crochet, S. and Petersen, C.C.H. (2016) Movement Initiation Signals in Mouse Whisker Motor Cortex. Neuron, 92, 1368-1382. https://doi.org/10.1016/j.neuron.2016.12.001

[81] Nathan, P.W. (1963) The Descending Respiratory Pathway in Man. Journal of Neurology, Neurosurgery and Psychiatry, 26, 487-499.

https://doi.org/10.1136/jnnp.26.6.487 
[82] Plum, F. (1970) Neurological Integration of Behavioural and Metabolic Control of Breathing. In: Porter, R., Ed., Breathing. Hering-Breuer Centenary Symposium, Churchill, London, 159-181. https://doi.org/10.1002/9780470715352.ch10

[83] Plassman, B.L. and Gandevia, S.C. (1989) Comparison of Human Motor Cortical Projections to Abdominal Muscles and Intrinsic Muscles of the Hand. Experimental Brain Research, 78, 301-308. https://doi.org/10.1007/BF00228901

[84] Macefield, G. and Gandevia, S.C. (1991) The Cortical Drive to Human Respiratory Muscles in the Awake State Assessed by Premotor Cerebral Potentials. Journal of Physiology, 439, 545-558. https://doi.org/10.1113/jphysiol.1991.sp018681

[85] Gandevia, S.C. and Rothwell, J.C. (1987) Activation of the Human Diaphragm from the Motor Cortex. Journal of Physiology, 384, 109-118.

[86] Gonella, J., Bouvier, M. and Blanquet, F. (1987) Extrinsic Nervous Control of Motility of Small and Large Intestines and Related Sphincters. Physiological Reviews, 67, No. 3. https://doi.org/10.1152/physrev.1987.67.3.902

[87] Netter, F.H. (1962) The Ciba Collection of Medical Illustrations. In: Oppenheimer, E.O., Ed., Digestive System, Lower Digestive Tract, Vol. 3, Ciba Pharmaceutical, New York, 243.

[88] Bywater, R.A.R. (1993) Activity Following Colonic Distension in Enteric Sensory Fibres Projecting to the Inferior Mesenteric Ganglion in the Guinea Pig. Journal of the Autonomic Nervous System, 46, 19-26.

[89] Messenger, J.P. and Furness, J.B. (1993) Distribution of Enteric Nerve Cells Projecting to the Superior and Inferior Mesenteric Ganglia of the Guinea-Pig. Cell and Tissue Research, 271, 333-339. https://doi.org/10.1007/BF00318620

[90] Altschuler, S.M., Ferenci, D.A., Lynn, R.B. and Miselis, R.R. (1991) Representation of the Cecum in Lateral Dorsal Motor Nucleus of the Vagus Nerve and Commissural Subnucleus of the Nucleus Tractus Solitarii in Rat. Journal of Comparative Neurology, 304, 261-274. https://doi.org/10.1002/cne.903040209

[91] Altschuler, S.M., Escardo, J., Lynn, R.B. and Miselis, R.R. (1993) The Central Organization of the Vagus Nerve Innervating the Colon of the Rat. Gastroenterology, 104, 502-509. https://doi.org/10.1016/0016-5085(93)90419-D

[92] Berthoud, H.R., Carlson, N.R. and Powley, T.L. (1991) Topography of Efferent Vagal Innervation of the Rat Gastrointestinal Tract. APS Journals, 260, R200-R207. https://doi.org/10.1152/ajpregu.1991.260.1.R200

[93] (2010) Yellow Emperor's Canon of Medicine. Volume 2, Spiritual Pivot, Wisdom of Chinese Culture Series. Chiao Liu (Canada) Publishing Trading Co. Ltd., Chief Editor: Ng Kin Sing, Hong Kong.

[94] Liu, P., Wang, P., Chen, G. and Liu, S.L. (2010) Theoretical Exploration and Clinical Application of the Lung and the Large Intestine Being Interior-Exteriorly Related. Journal of Liaoning University of Traditional Chinese Medicine, 12, 15-17.

[95] Yang, S., Wang, X., Yang, X., Jing, S., Zhu, L. and Wang, S. (2012) Comparison between Effects of Traditional Chinese Medicine Treatment from the Lung and Treatment from the Intestine on the Level of VIP in Rats with Crohn's Disease. World Science and Technology (Modernization of Traditional Chinese Medicine and Material Medical), 14, 1841-1847.

[96] Smith, J.C., Abdala, A.P.L., Koizumi, H., Rybak, I.A. and Paton, J.F.R. (2007) Spatial and Functional Architecture of the Mammalian Brainstem Respiratory Network: A Hierarchy of Three Oscillatory Mechanisms. Journal of Neurophysiology, 98, 3370-3387. https://doi.org/10.1152/jn.00985.2007 
[97] Smith, J.C., Abdala, A.P.L., Rybak, I.A. and Paton, J.F.R. (2009) Structural and Functional Architecture of Respiratory Networks in the Mammalian Brainstem. Philosophical Transactions of the Royal Society B, 364, 2577-2587. https://doi.org/10.1098/rstb.2009.0081

[98] Smith, J.C., Morrison, D.E., Ellenberger, H.H., Otto, M.R. and Feldman, J.L. (1989) Brainstem Projections to the Major Respiratory Neuron Populations in the Medulla of the Cat. Journal of Comparative Neurology, 281, 69-96. https://doi.org/10.1002/cne.902810107

[99] Yates, B.J. and Millert, A.D. (1998) Physiological Evidence that the Vestibular System Participates in Autonomic and Respiratory Control. Journal of Vestibular Research, 8, 17-25. https://doi.org/10.1016/S0957-4271(97)00035-9

[100] Ghali, M.G.Z. (2017) The Bulbospinal Network Controlling the Phrenic Motor System: Laterality and Course of Descending Projections. Neuroscience Research, 121, 7-17. https://doi.org/10.1016/j.neures.2017.03.004

[101] Stornetta, R.L. (2009) Neurochemistry of Bulbospinal Presympathetic Neurons of the Medulla Oblongata. Journal of Chemical Neuroanatomy, 38, 222-230. https://doi.org/10.1016/j.jchemneu.2009.07.005

[102] Guyenet, P.G. (2014) Regulation of Breathing and Autonomic Outflows by Chemoreceptors. Comprehensive Physiology, 4, 1511-1562. https://doi.org/10.1002/cphy.c140004

[103] Smith, J.C., Greer, J.J., Liu, G.S. and Feldman, J.L. (1990) Neural Mechanisms Generating Respiratory Pattern in Mammalian Brain Stem-Spinal Cord in Vitro I. Spatiotemporal Patterns of Motor and Medullary Neuron Activity. Journal of Neurophysiology, 64, No. 4. https://doi.org/10.1152/jn.1990.64.4.1149

[104] Vinit, S. and Kastner, A. (2009) Descending Bulbospinal Pathways and Recovery of Respiratory Motor Function Following Spinal Cord Injury. Respiratory Physiology and Neurobiology, 169, 115-122. https://doi.org/10.1016/j.resp.2009.08.004

[105] Dobbins, E.G. and Feldman, J.L. (1994) Brainstem Network Controlling Descending Drive to Phrenic Motoneurons in Rat. Journal of Comparative Neurology, 347, 64-68. https://doi.org/10.1002/cne.903470106

[106] Arnold, A.C., Shaltout, H.A., Patricia, E., Gallagher, P.E. and Diz, D.I. (2009) Leptin Impairs Cardiovagal Baroreflex Function at the Level of the Solitary Tract Nucleus. Hypertension, 54, 1001-1008. https://doi.org/10.1161/HYPERTENSIONAHA.109.138065

[107] Evans, K.C., Dougherty, D.D., Schmid, A.M., Scannell, E., McCallister, A., Benson, H., Dusek, J.A. and Lazar, S.W. (2009) Modulation of Spontaneous Breathing via Limbic/Paralimbic-Bulbar Circuitry: An Event Related fMRI Study. Neuroimage, 47, 961-971. https://doi.org/10.1016/j.neuroimage.2009.05.025

[108] Evans, K.C. (2010) Cortico-Limbic Circuitry and the Airways: Insights from Functional Neuroimaging of Respiratory Afferents and Efferents. Biological Psychology, 84, 13-25. https://doi.org/10.1016/j.biopsycho.2010.02.005

[109] McKay, L.C., Adams, L., Frackowiak, R.S. and Corfield, D.R. (2008) A Bilateral Cortico-Bulbar Network Associated with Breath Holding in Humans, Determined by Functional Magnetic Resonance Imaging. Neuroimage, 40, 1824-1832. https://doi.org/10.1016/j.neuroimage.2008.01.058

[110] Kato, A., Takahashi, K. and Homma, I. (2017) Relationships between Trait and Respiratory Parameters during Quiet Breathing in Normal Subjects. The Journal of Physiological Sciences, 68, 369-376. https://doi.org/10.1007/s12576-017-0539-7

[111] Wang, L.L., Jia, W.H., Yang, J., Liu, R. and Ma, Y.D. (2013) Acupuncture, Cupping 
Treatment of Chronic Obstructive Pulmonary Disease Research Progress. Hebei Journal of Traditional Chinese Medicine, 35, 1095.

[112] Qiu, W.W. (1999) Lei Que is the Main Acupoint for Cold Phlegm Treatment. Shanghai Journal of Acupuncture and Moxibustion, 18, 12.

[113] Wan, W.R. (2006) Acupuncture Treatment of Chronic Obstructive Pulmonary Disease in 36 Cases. Chinese Acupuncture \& Moxibustion, 26, 672.

[114] Li, H.Z., Qin, X.Y., Shao, S.J. and Hua, J.S. (2015) Research Situation on the Treatment of Chronic Obstructive Pulmonary Disease by Acupuncture and Moxibustion. China Journal of Chinese Medicine, 30, 484-486.

[115] Sato, A. (1997) Neural Mechanisms of Autonomic Responses Elicited by Somatic Sensory Stimulation. Neuroscience and Behavioral Physiology, 27, 610-621. https://doi.org/10.1007/BF02463910

[116] Ip, M.S.M., Lam, B., Chan, L.Y., Zheng, L., Tsang, K.W.T., Fung, P.C.W. and Lam, W.K. (2000) Circulating Nitric Oxide Is Suppressed in Obstructive Sleep Apnea and Is Reversed by Nasal Continuous Positive Airway Pressure. American Journal of Respiratory and Critical Care Medicine, 162, 2166-2171.

https://doi.org/10.1164/ajrccm.162.6.2002126

[117] Tong, F., Guo, Y.M., He, Y., Li, G.Y., Chen, F. and Yao, H. (2014) Regulatory Effects of Acupuncture on Exercise Tolerance in Patients with Chronic Obstructive Pulmonary Disease at Stable Phase: A Randomized Controlled Trial. Chinese Acupuncture \& Moxibustion, 34, 846-850.

[118] Fung, P.C.W. and Kong, R.K.C. (2017) The Heat Shock Protein Story-From Taking mTORC1,2 and Heat Shock Protein Inhibitors as Therapeutic Measures for Treating Cancers to Development of Cancer Vaccines. Journal of Cancer Therapy, 8, Article ID: 80657. https://doi.org/10.4236/jct.2017.811086

[119] Zhou, Y.L., Su, C.G., Liu, S.F., Jin, X.Y., Duan, Y.L., Chen, X.Y., Zhao, S.H., Wang, Q.L. and Dang, C.L. (2016) Resting-State Functional Magnetic Resonance Imaging; Hand Taiyin Lung Channel; Amplitude of Low Frequency Fluctuation; Brain Function Network. CJITWM, 36, 553-558. 